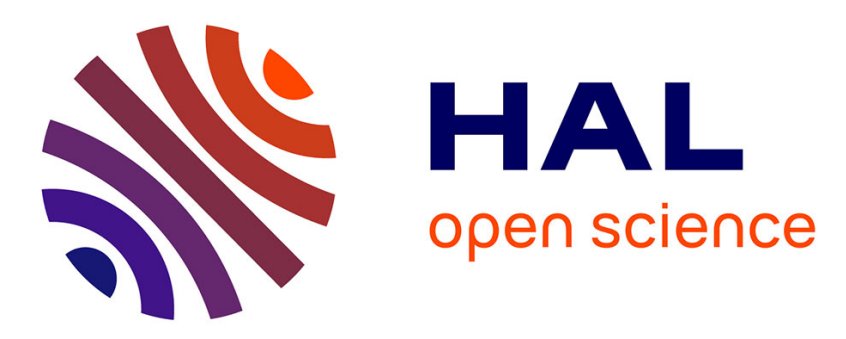

\title{
Multi-objective energy management for fuel cell electric vehicles using online-learning enhanced Markov speed predictor
}

\author{
Yang Zhou, Alexandre Ravey, Marie-Cécile Péra
}

\section{- To cite this version:}

Yang Zhou, Alexandre Ravey, Marie-Cécile Péra. Multi-objective energy management for fuel cell electric vehicles using online-learning enhanced Markov speed predictor. Energy Conversion and Management, 2020, 213, pp.112821 (18). hal-02993892

\section{HAL Id: hal-02993892 \\ https://hal.science/hal-02993892}

Submitted on 7 Nov 2020

HAL is a multi-disciplinary open access archive for the deposit and dissemination of scientific research documents, whether they are published or not. The documents may come from teaching and research institutions in France or abroad, or from public or private research centers.
L'archive ouverte pluridisciplinaire HAL, est destinée au dépôt et à la diffusion de documents scientifiques de niveau recherche, publiés ou non, émanant des établissements d'enseignement et de recherche français ou étrangers, des laboratoires publics ou privés. 


\title{
Multi-objective Energy Management for Fuel Cell Electric Vehicles using Online-Learning Enhanced Markov Speed Predictor
}

\author{
Yang Zhou*, Alexandre Ravey, Marie-Cécile Péra \\ FEMTO-ST (UMR CNRS 6174), FCLAB (FR CNRS 3539), Univ. Bourgogne Franche-Comté, UTBM \\ Rue Thierry Mieg, BELFORT, FRANCE. \\ yang.zhou@utbm.fr ; alexandre.ravey@utbm.fr ; marie-cecile.pera@univ-fcomte.fr. \\ * Corresponding Author.
}

\begin{abstract}
As one of promising solutions towards future cleaner transportation, fuel cell electric vehicles have been widely regarded as an attractive technology in both academia and industry. To enhance the vehicle's operation efficiency, this paper proposes a multi-criteria power allocation strategy for a fuel cell/batterybased plug-in hybrid electric vehicle. Firstly, an adaptive online-learning enhanced Markov velocityforecast approach is proposed. Its predictive behaviors can be adjusted accordingly under various driving scenarios through the real-time-identified transition probability matrices. Subsequently, based only on the previewed trip duration information and the speed prediction results, a state-of-charge (SOC) reference planning approach is designed to guide the allocation of battery energy. Combining with the velocity-forecast results and the reference SoC, model predictive control derives the optimal powerallocation decision through minimizing the multi-purpose objective function in a finite time horizon. It has been verified that (1) the presented power allocation strategy can reduce over $12.05 \% \mathrm{H} 2$ consumption and over $94.40 \%$ fuel cell power spikes against the commonly used ChargeDepleting/Charge-Sustaining strategy; (2) despite the existence of mission time estimation errors, the presented control strategy could still bring performance enhancement over the benchmark strategy, thus demonstrating its feasibility for real-world implementations.
\end{abstract}

Key Words: Energy Management Strategy, Fuel Cell, Plug-in Hybrid Electric Vehicles, Speed Forecasting Technique, State-of-Charge Reference Generation. 


\begin{tabular}{|c|c|c|c|}
\hline PHEV & Plug-in Hybrid Electric Vehicle & SYMBOLS & \\
\hline ICE & Internal Combustion Engine & $P_{d}$ & DC bus Power demand \\
\hline FCPHEV & Fuel Cell Plug-in Hybrid Electric Vehicle & $P_{f c}$ & PEMFC system net power \\
\hline PEMFC & Proton Exchange Membrane Fuel Cell & $P_{b}$ & Battery Power \\
\hline FCS & Fuel Cell System & $m_{\mathrm{H}_{2}}$ & Actual Hydrogen Mass Consumption \\
\hline EMS & Energy Management Strategy & $m_{e q u} H_{2}$ & Equivalent Hydrogen Mass Consumption \\
\hline CD-CS & Charge-Depleting Charge-Sustaining & SoC & Battery State-of-Charge \\
\hline SoC & State-of-charge & $U_{d c}$ & DC Bus Voltage \\
\hline DP & Dynamic Programming & $\pi_{1}, \pi_{2}, \pi_{3}$ & MPC Penalty Factors \\
\hline GA & Genetic Algorithm & $H_{P}$ & Prediction Horizon \\
\hline PMP & Pontryagin's Minimum Principle & $T_{l}$ & $l$-step Transition Probability Matrix \\
\hline ECMS & Equivalent Consumption Minimization Strategy & $s$ & Total Number of Markov State \\
\hline MPC & Model Predictive Control & $\mu$ & Forgetting Coefficient \\
\hline DPR & Driving Pattern Recognition & $D_{\mu}$ & Markov Chain Effective Memory Depth \\
\hline GPS & Global Positioning System & $\overline{\left|\Delta P_{f c}\right|}$ & Root-mean-square of FC Power Transients \\
\hline ITS & Intelligent Transportation System & $\operatorname{SoC}_{0}$ & Initial Battery SoC \\
\hline MC & Markov Chain & $\operatorname{SoC}_{N}$ & Final Battery SoC \\
\hline BPNN & Back Propagation Neural Network & $T_{\text {trip }}$ & Estimated Trip Duration \\
\hline TPM & Transition Probability Matrix & $\alpha$ & Adjusting Factor \\
\hline PEMS & Predictive Energy Management Strategy & $r_{S o C} / r_{S o C}^{\prime}$ & Reference/Actual SoC Depletion Rate \\
\hline MPC & Model Predictive Control & $\overline{R M S E}$ & Average Root Mean Square Error \\
\hline \multirow[t]{2}{*}{ QP } & Quadratic Programming & $v_{\text {ave }}$ & Average of Forecasted Speed Sequence \\
\hline & & $v_{s t d}$ & Standard Deviation of Forecasted Speed Sequence \\
\hline
\end{tabular}

\section{Introduction}

For mitigating the dependency on fossil fuels, plug-in hybrid electric vehicles (PHEV) are widely considered as one of key technologies towards future cleaner mobility [1]. Owing to its zero-emission property, the fuel cell system (FCS) is capable of directly converting chemical energy into deliverable electrical energy, making it the ideal substitution to internal combustion engines (ICE) [2]. In accordance with such trend, fuel cell/battery-based PHEV (abbreviated as FCPHEV) has attracted substantial research attentions in green transport field most recently [3]. However, numerous un-well-solved issues, like the fuel cell durability and the shortage of hydrogen refueling infrastructures, significantly hinder the commercialization of fuel cell vehicles [4]. Therefore, to reduce the operation costs of FCPHEVs, a robust energy management strategy (EMS), which can achieve the reliable energy distribution by regulating the output behaviors of multiple energy sources within the hybrid powertrain, should be further investigated.

\subsection{Literature review}

The power splitting strategies for PHEVs can be cataloged into Charge-Depleting/Charge-Sustaining (CD-CS) strategy and blended strategy. The principle of CD-CS strategy is to operate the vehicle as a 
pure EV until the State-of-Charge (SoC) of power battery reaches a preset lower threshold. Afterwards, the primary energy source switches on to maintain the SoC level [5]. However, the predefined control parameters in CD-CS strategy cannot fully ensure the performance optimality under various driving conditions, especially when the trip length exceeds the all-electric-range of PHEVs. Alternatively, several blended EMSs using dynamic programming (DP) [6], [7], genetic algorithm (GA) [8], [9] and Pontryagin's minimum principle (PMP) [10], [11] can acquire the global optimal performance by minimizing the predefined objective functions. However, these strategies can only be deemed as the offline benchmarks due to the requirement on the complete route information as well as the unavoidable huge computation costs.

As a substitute solution, real-time optimization-based strategies become appealing to researchers, including equivalent consumption minimization strategy (ECMS) [12], [13] and model predictive control (MPC) [14]-[19]. As the decision maker within the EMS framework for PHEVs, MPC is capable of anticipating future system behaviors and takes control actions accordingly by optimizing the performance index in a finite time horizon [14]. To be specific, the performance of MPC-based EMS is largely dependent on two essential factors. The first one is battery SoC reference trajectory. In fact, the fuel economy of PHEVs is closely related to the way of battery energy usage during a trip. Therefore, an explicit SoC reference trajectory is indispensable as a guidance for planning battery energy distribution to approximate the global optimality [15]. The second one is the forecasted speed profile over each rolling optimization horizon. In MPC control framework, the upcoming vehicle speed is often regarded as the disturbances and the quality of speed prediction directly affects the MPC performance [16]. However, under realistic driving conditions, the vehicle's velocity could be affected by many uncertainties (e.g. the stochastic distribution of traffic lights and the unexpected pedestrian movement, etc.), and thus is very hard to forecast.

Consequently, to provide with accurate reference and predictive information for MPC decision-making, it is meaningful to investigate the battery SoC reference generation methods and the vehicle speed forecasting techniques, which is the major research focus of this paper. In fact, large number of researches has been conducted on these topics [20]-[26]: 
Generally, the methods for SoC reference generation can be roughly classified into three types. The first type is based on linear SoC reference model. With the estimated trip length [20] (or duration [14]), the reference SoC is designed to linearly decline from the initial (maximum) value to the terminal (minimum) one. Moreover, to improve the rationality in battery energy planning, authors in [15], [16] have added some adaptation laws to the original linear model, making the SoC declining rate change with the realistic driving conditions, thus leading to better EMS performance against the original linear model. The second type of SoC reference planning method takes advantage of the real-time updated route-based information from intelligent transportation system (ITS) or global positioning system (GPS) [21]-[23]. For instance, in [21], DP extracts the optimal SoC traces from the real-time traffic flow speed profiles. Afterwards, the obtained SoC references are given to the MPC controller for guiding the energy distribution. In this way, nearly $95 \%$ fuel optimality compared to DP benchmark is achieved by the proposed hierarchical EMS. The third type of method benefits from data-driven approaches [18], [24][26]. For instance, based on the abundant historical driving database of plug-in hybrid electric buses, a multi-variant regression model is developed to generate the $\mathrm{SoC}$ reference trajectories [18], where the fitting coefficients are obtained from the DP-optimized SoC traces. In this way, compared with a rulebased benchmark, the proposed strategy can improve fuel economy by $42.46 \%$.

To characterize the upcoming velocity trajectories over each preview horizon, two types of data-driven methodologies are widely employed in previous researches, namely the Markov Chain (MC) models [14], [15], [18] and neural network (NN) models [16], [21], [23]. For example, authors in [15] have built a multi-step MC velocity predictor based on real bus driving database, which outperforms the back propagation NN (BPNN) predictor in terms of the computation efficiency and the overall forecasting precision. Besides, a deep neural network based speed-forecast method is reported in [23], which can respectively enhance the forecast precision by $26.8 \%$ and $22.4 \%$ compared to the traditional $\mathrm{MC}$ and BPNN predictor.

\subsection{Motivations and Innovations}

Despite large amount of MPC-based EMSs have been devised for PHEVs in previous researches, there still exists plenty room for improvement in following aspects: 
- In our previous works, an Elman NN predictor [17] and a fuzzy C-means enhanced Markov predictor [19] are built using the offline historical driving database. Nevertheless, their prediction quality would be greatly challenged if the discrepancy between the realistic driving scenarios and the historical ones were significant [27]. To compensate for this defect, the online learning mechanism should be introduced to update the speed predictors and help them adapt to new driving scenarios in a stepwise manner. Through such adaptation law, the precision and reliability of the speed predictors in our previous works can be further enhanced.

- The single SoC declining rate of linear SoC reference model [14] may be improper for realistic cycles with changeable driving patterns. To overcome this defect, we propose an adaptive method for SoC reference planning in our previous work [19], but its effectiveness is only verified under urban driving scenario. Besides, the requirement on real-time traffic information and the bulky computational burden greatly hinder the real application of telematics-based approaches [21]. Hence, an integrable solution for SoC reference generation should be further explored, which can effectively guide battery energy depletion in face of the changes in driving patterns.

- H2 consumption saving and FCS lifetime prolongation by avoid harsh transients are two essential EMS objectives in our previous work [17]. However, the SoC reference estimation errors caused by future driving uncertainties would compromise the EMS performance. Hence, how to ensure fuel economy, FCS durability and SoC regulation capacity, while compensating for the EMS performance losses caused by SoC reference estimation errors still needs to be intensively studied.

To compensate for these deficiencies, a power allocation strategy considering velocity prediction is developed in this study, which contains the following contributions:

- An adaptive online-learning enhanced Markov speed forecasting method is proposed. Two features make the proposed method distinct from the ones in our previous works: (i) without using offlinedriving database, the self-learning MC is capable of stepwise renewing its transition probability matrices (TPM) through the real-time obtained driving samples; (ii) with the real-time renewed TPMs, the proposed method can adjust its predictive behaviors towards different driving patterns, thus enhancing the prediction robustness. 
- Only based on the estimated trip duration information, a SoC reference generator is developed, which can be integrated into the EMS and is capable of regulating the SoC declining ratio in multiple driving scenarios with the help of the real-time updated speed forecasting results.

- Combining the velocity-forecast results and the reference SoC traces, a multi-criterion MPC-based EMS is devised, aiming at enhancing the FCS's operation efficiency and prolonging the FCS's service time, while compensating for the potential EMS performance losses caused by SoC reference estimation errors.

\subsection{Article overview}

The sequel of this article is sketched as below. The studied vehicle model is established in section II. The design of the self-learning MC prediction approach, the SoC reference planning approach and the MPC control strategy are detailed in Section III. The devised strategy is thoroughly validated in section IV. Key findings and future research directions are summarized in section V.

\section{Modelling of a middle-sized vehicle}

\subsection{Vehicle dynamics and powertrain topology}

From the vehicular simulator ADVISOR, a middle-sized car model is picked for control strategy development. As depicted in Fig. 1(a), the PEMFC and battery work cooperatively to response the power demand from the electric machine, where PEMFC is attached to the DC bus through a DC/DC converter and battery is straight attached to the DC bus. Benefiting from an available component-sizing configuration for middle-sized FCPHEVs in [16], the specifications of the studied hybrid powertrain are given in TABLE I. In such powertrain topology, only the FCS output power can be actively controlled $[28]$.

In addition, the vehicular power requirement in motion as a function of its weight $M$ and desired speed $v$ can be expressed by (1) [29]. Please note the gravitational acceleration $g=9.81 \mathrm{~m} / \mathrm{s}^{2}$. Besides, as a horizontal road-vehicle configuration (see Fig. 1(b)) is used for calculating the external power demands, the angle of road inclination $\theta$ takes zero. Meanwhile, the net power of PEMFC system $\left(P_{f c}\right)$ and battery power $\left(P_{b}\right)$ together response to the equivalent power demand ( $\left.P_{d}\right)$ on DC bus, as indicated in (2) [30]. 
151

152

153

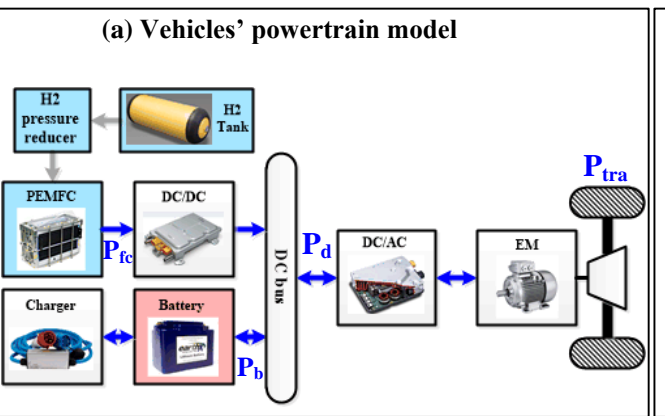

(b) Vehicles' dynamics on horizontal road

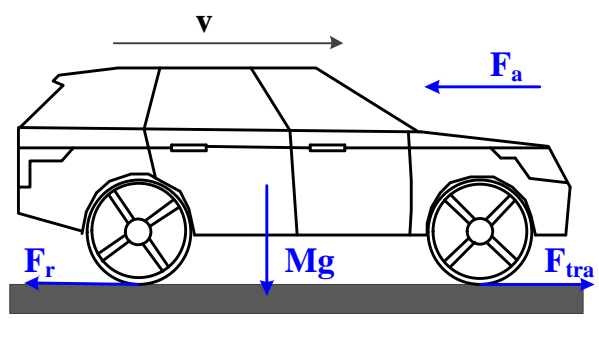

Fig. 1. Schematic diagram of (a) hybrid powertrain structure and (b) forces on a vehicle in motion.

$P_{t r a}=v \cdot(\underbrace{c_{r} M g \cos (\theta)}_{\mathbf{F}_{\mathbf{r}}}+\underbrace{\frac{1}{2} \rho_{a i r} S_{f} c_{d} v^{2}}_{\mathbf{F}_{\mathbf{a}}}+M \dot{v})$

$$
P_{d}=\frac{P_{t r a}}{\eta_{t r a} \cdot \eta_{D C / A C} \cdot \eta_{E M}}=P_{b}+P_{f c} \cdot \eta_{D C / \mathrm{DC}}
$$

155

Where $c_{r}$ represents the rolling resistance coefficient, $\rho_{\text {air }}$ the air density, $S_{f}$ the area of front surface, $c_{\boldsymbol{d}}$ the aerodynamic drag coefficient, $\eta_{\text {tra }}$ the driveline efficiency, $\eta_{D C / A C}$ and $\eta_{D C / D C}$ the power converters' efficiencies.

TABLE I. Vehicular Powertrain Specifications

\begin{tabular}{c|c|c|c}
\hline Component & Description & Value & Unit \\
\hline \multirow{3}{*}{ Sedan Structural } & Curb Weight & 1360 & $\mathrm{~kg}$ \\
Parameters from & Frontal Area & 1.746 & $\mathrm{~m}^{2}$ \\
ADVISOR & Density of Air & 1.21 & $\mathrm{~kg} / \mathrm{m}^{3}$ \\
& Coefficient of Aerodynamic drag & 0.3 & $\mathrm{~N} / \mathrm{A}$ \\
& Coefficient of Rolling Resistance & 0.0135 & $\mathrm{~N} / \mathrm{A}$ \\
& Efficiency for driveline & 0.91 & $\mathrm{~N} / \mathrm{A}$ \\
\hline \multirow{2}{*}{ PEMFC System } & Peaking power & 30 & $\mathrm{~kW}$ \\
& Peaking Efficiency & $50.3 \%$ & $\mathrm{~N} / \mathrm{A}$ \\
\hline Lithium-ion battery & Nominal Energy Capacity & 12.8 & $\mathrm{kWh}$ \\
\hline \multirow{2}{*}{ Traction Motor } & Allowable max. Power & 75 & $\mathrm{~kW}$ \\
& Allowable max. Torque & 271 & $\mathrm{~N} \cdot \mathrm{m}$ \\
& Allowable max. Speed & 10000 & $\mathrm{rpm}$ \\
\hline Power Converter & DC - DC & 0.90 & $\mathrm{~N} / \mathrm{A}$ \\
Efficiency & DC - AC & 0.95 & $\mathrm{~N} / \mathrm{A}$ \\
\hline
\end{tabular}

\subsection{Fuel Cell Model}

As one of widely used fuel cells in automotive industry, the PEMFC is embedded in the studied powertrain. Under the specific working conditions (e.g. humidity, partial pressure etc.), the cell voltage can be written as a function of the current density [29]. Additionally, given the lower heating value of $\mathrm{H} 2\left(\mathrm{LHV}_{\mathrm{H}_{2}}\right.$ in MJ / kg $)$, the mass of hydrogen $\left(\mathrm{m}_{\mathrm{H}_{2}}\right)$ utilized over a trip is derived by [31]: 
$m_{H_{2}}=\int_{0}^{t} \frac{P_{f c}(t)}{\eta_{F C S} \cdot \mathrm{LHV}_{\mathrm{H}_{2}}} d t$

Note $\eta_{F C S}$ is the efficiency of fuel cell system. Actually, two major components can be found within a typical PEMFC system, including the fuel cell stack (which transforms the hydrogen energy into the electricity power through chemical reactions) and the auxiliary devices (which guarantee the normal operation of fuel cell stack). Consequently, $\eta_{F C S}$ can be defined by:

$\eta_{F C S}=\frac{P_{f c}}{P_{\text {chemical }}}=\frac{P_{\text {stack }}-P_{A U X}}{P_{\text {chemical }}}$

Where $P_{\text {stack }}$ represents the fuel cell stack output electrical power, $P_{A U X}$ the power dissipated in the auxiliaries and $P_{\text {Chemical }}$ the energy flux contained in reactants [30]. Hence, $P_{f c}$ represents the net power output from PEMFC system. Specifically, Fig. 1(c) depicts the relationship between $P_{f c}$ and $\eta_{F C S}$ in the studied powertrain. The system peaking efficiency $\eta_{\max }=50.3 \%$ is associated with the most efficient fuel cell power point $P_{\eta}^{\max }$. Besides, $P_{f_{c}} \in\left[P_{\eta}^{L O W}, P_{\eta}^{H I G H}\right]$ defines the high efficiency area $\left(\eta_{F C S} \geq 47.0 \%\right)$ of the FCS.

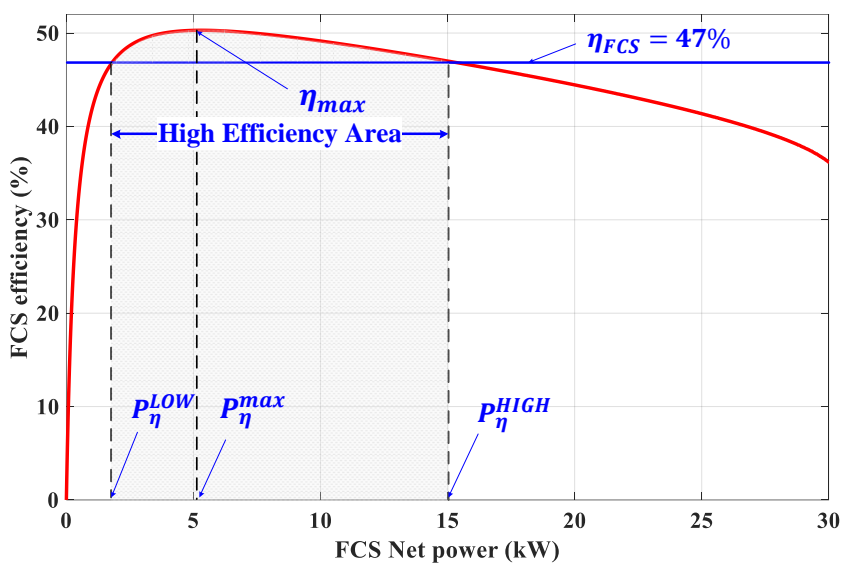

Fig. 1(c). The relationship between FCS net power $\left(P_{f c}\right)$ and FCS efficiency $\left(\eta_{F C S}\right)$.

\subsection{Battery Model}

As shown in Fig. 1(d), the internal-resistance model is adopted in this work to characterize the lithiumion battery. In addition, let $I_{b}$ denotes the battery current, $R_{b}$ the battery resistance, $Q_{b}$ the nominal 
$S o C(\mathrm{t})=S o C_{0}-\frac{\int_{0}^{t} \eta_{b} \cdot I_{b}(\mathrm{t}) \mathrm{dt}}{Q_{b}}$

$I_{b}=\frac{U_{o c}(S o C)-\sqrt{U_{o c}(S o C)^{2}-4 \cdot R_{b}(S o C) \cdot \mathrm{P}_{b}}}{2 \cdot R_{b}(S o C)}$

$U_{d c}=U_{o c}(S o C)-I_{b} \cdot R_{b}(S o C)$

Where $S o C_{0}$ denotes the initial SoC state. It should be mentioned that the values for $U_{o c}$ and $R_{b}$ of a battery cell vary with its SoC. To characterize such nonlinear relationship, an experimentally validated lithium-ion battery model is picked from the database of vehicular simulator ADVISOR [32]. As illustrated in Fig. 1(e), when $0.3 \leq \mathrm{SoC} \leq 0.9$, the OCV of battery cell declines linearly and the variation of internal resistance is insignificant. Hence, in light of battery working safety and efficiency issues, restricting SoC in its normal operating region is commonly recommended.
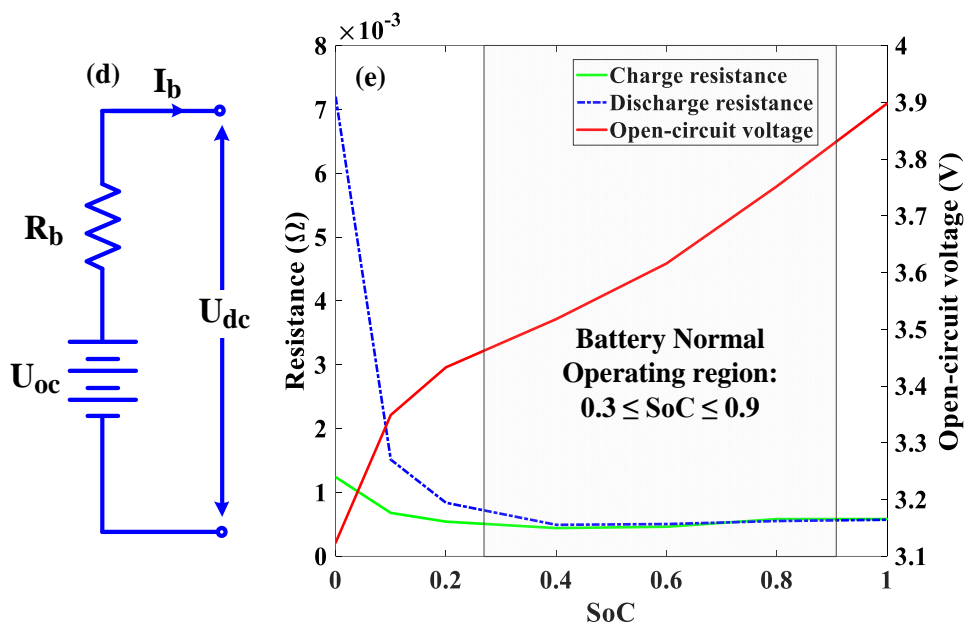

Fig. 1. (d) Battery internal-resistance model and (e) battery cell parameters variation with SoC.

192

193

\subsection{Electric Machine Model}

Electric machine (EM) is the provider of vehicle propulsion power. According to the maximum power and torque demands required by the driving cycles, a $75-\mathrm{kW}$ AC induction motor is picked as the computation model in this work. Please note this experimentally validated EM model is selected form ADVISOR database, whose permissible torque and rotation speed ranges are $T_{\text {motor }} \in$ $[-271,271] \mathrm{N} \cdot \mathrm{m}$ and $\omega_{\text {motor }} \in[0,10000] \mathrm{rpm}$, respectively. Besides, the EM efficiency 
$\eta_{\text {motor }}$ changes with the motor working states specified by $T_{\text {motor }}$ and $\omega_{\text {motor }}$. Consequently, given the 199 desired vehicular speed and torque requests, the motor working efficiency can be looked up through the efficiency map (Fig. 1(f)).

Efficiency Map of the Electrical Machine

Fig. 1(f). Motor efficiency as a function of $T_{\text {motor }}$ and $\omega_{\text {motor }}$.

\section{Predictive Energy Management Strategy Design}

Fig. 2 schematically presents the control framework for the devised predictive energy management strategy. In the supervisory level, the self-learning MC predictor can forecast the speed profiles with the real-time updated TPM group. Afterwards, the declining rate of battery $\mathrm{SoC}$ is regulated according to the partial trip information and speed forecasting results. In the rolling optimization level, combining the velocity-forecast results, the reference SoC traces and the current vehicle states, MPC derives the optimal control action through minimizing the multi-purpose objective function at each time step. The development of PEMS is thoroughly illustrated afterwards. 


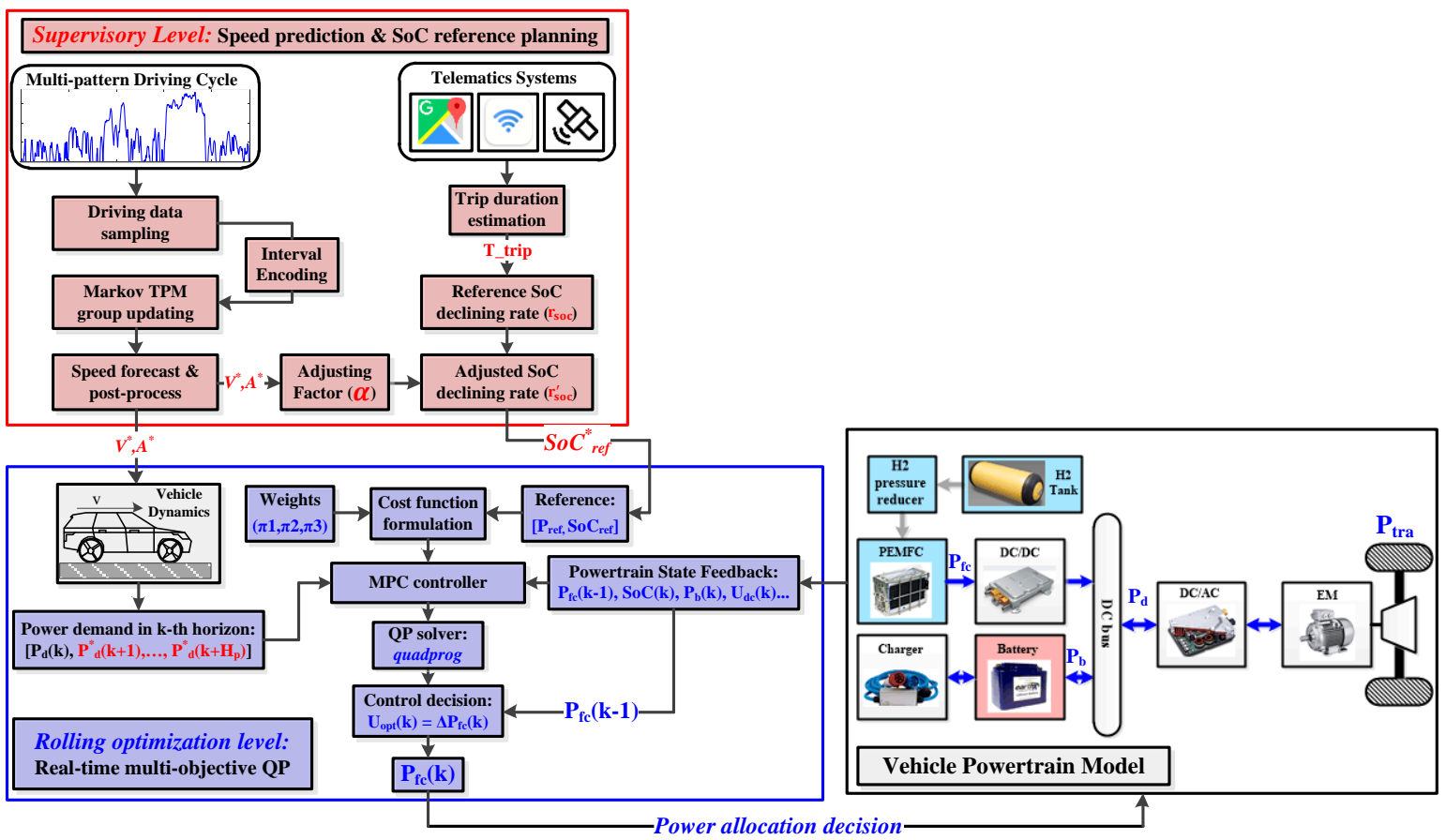

Fig. 2. Control framework for the devised predictive energy management strategy.

\section{3.1. Speed Forecasting Technique}

214 The quality of velocity prediction would largely affect the MPC decision-making process. To enhance the forecast precision under rapid-changing driving scenarios, the design of an adaptive self-learning enhanced Markov speed predictor is detailed in the remaining part of subsection 3.1.

\subsubsection{Markov Chain and online-learning technique}

The future acceleration distribution is taken as a stochastic process, which is modeled by Markov Chain. Under the interval-encoding framework [33], the continuous acceleration domain is discretized by numerous disjoint intervals $I_{j}, j \in\{1, \ldots, \mathrm{s}\}$, where every interval midpoint is tagged by a single Markov state, marked as $a_{j} \in I_{j}$. Subsequently, a countable set $X_{\mathrm{a}}=\left\{a_{1}, \ldots, a_{s}\right\}$ containing all feasible 222 acceleration states defines the state space of Markov model. For multi-step prediction purpose, a TPM 223 group $\mathrm{T}_{\mathbf{G}}=\left\{T_{1}, \ldots, T_{\mathrm{H}_{\mathbf{p}}}\right\}$ should be built. Note the $l$-th TPM in $\mathrm{T}_{\mathbf{G}}$ is an s-order square matrix denoting 224 the $l$-step $\left(\boldsymbol{l} \in\left\{1, \ldots, \mathrm{H}_{\mathbf{p}}\right\}, \mathrm{H}_{\mathrm{p}}\right.$ is the prediction horizon) ahead probability distribution. Its element in the $225 i$-th row and $j$-th column, denoted as $\left[T_{l}\right]_{i j}$, indicates the probability of a state transition from $a_{i}$ to $a_{j}, i, j \in\{1, \ldots, \mathrm{s}\}$, where the value of $\left[T_{l}\right]_{i j}$ can be derived by Eq. (6). 
228

229

Please note $\operatorname{Num}_{i j}^{l}$ and $\mathrm{Num}_{o i}^{l}$ are the numbers of Markov state transition, with the superscript $l$ denoting the time step and the subscript denoting the indices of transition incidents ( $i j$ for the transitions from $a_{i}$ to $a_{j}$, whereas oi for the transitions originating from $a_{i}$ ).

To estimate the TPM group through the online measurements, the state transition number Num is substituted to the state transition frequency Fre. Consequently, the transition probability estimation model (6) can be rewritten as [33]:

$\left[T_{l}(L)\right]_{i j} \approx \frac{\operatorname{Num}_{i j}^{l}(L) / L}{\operatorname{Num}_{o i}^{l}(L) / L}=\frac{\operatorname{Fre}_{i j}^{l}(L)}{\operatorname{Fre}_{o i}^{l}(L)}$

$\operatorname{Fre}_{i j}^{l}(L)=\operatorname{Num}_{i j}^{l}(L) / L=\frac{1}{L} \sum_{t=1}^{L}$ flag $_{i j}^{l}(\mathrm{t})$

$\operatorname{Fre}_{o i}^{l}(L)=\operatorname{Num}_{o i}^{l}(L) / L=\frac{1}{L} \sum_{t=1}^{L} \operatorname{flag}_{o i}^{l}(\mathrm{t})$

Where $L$ denotes the observation length. Moreover, flag indicates the occurrence of related transition incidents. For instance, at time step $t(t \in[1, L])$, flag $_{i j}^{l}(\mathrm{t})=1$ or flag ${ }_{o i}^{l}(\mathrm{t})=1$ only when the related state transition incidents happen, where flag ${ }_{o i}^{l}(\mathrm{t})=\sum_{j=1}^{s}$ flag $_{i j}^{l}(\mathrm{t})$. If the related transition incidents do not happen, they both equal to zero. Moreover, the transition frequency $\operatorname{Fre}_{i j}^{l}(L)$ and $\operatorname{Fre}_{o i}^{l}(L)$ can be expanded in the following recursive form:

$$
\begin{aligned}
\operatorname{Fre}_{i j}^{l}(L) & =\frac{1}{L} \sum_{t=1}^{L} \operatorname{flag}_{i j}^{l}(\mathrm{t})=\frac{1}{L} \cdot\left[(L-1) \operatorname{Fre}_{i j}^{l}(L-1)+\operatorname{flag}_{i j}^{l}(L)\right] \\
& =\operatorname{Fre}_{i j}^{l}(L-1)+\frac{1}{L} \cdot\left[\operatorname{flag}_{i j}^{l}(L)-\operatorname{Fre}_{i j}^{l}(L-1)\right] \\
& \approx \operatorname{Fre}_{i j}^{l}(L-1)+\mu \cdot\left[\operatorname{flag}_{i j}^{l}(L)-\operatorname{Fre}_{i j}^{l}(L-1)\right] \\
\operatorname{Fre}_{o i}^{l}(L) & =\frac{1}{L} \sum_{t=1}^{L} \operatorname{flag}_{o i}^{l}(\mathrm{t})=\frac{1}{L} \cdot\left[(L-1) \operatorname{Fre}_{o i}^{l}(L-1)+\operatorname{flag}_{o i}^{l}(L)\right] \\
& =\operatorname{Fre}_{o i}^{l}(L-1)+\frac{1}{L} \cdot\left[\operatorname{flag}_{o i}^{l}(L)-\operatorname{Fre}_{o i}^{l}(L-1)\right] \\
& \approx \operatorname{Fre}_{o i}^{l}(L-1)+\mu \cdot\left[\operatorname{flag}_{o i}^{l}(L)-\operatorname{Fre}_{o i}^{l}(L-1)\right]
\end{aligned}
$$


Furthermore, the forgetting coefficient $\boldsymbol{\mu}(0<\mu<1)$ is introduced in $(7 \mathrm{~d})$ and (7e), which is equivalent to erasing the impact of older measurements through exponentially decreasing their weights. Hence, the probability $\left[T_{l}(L)\right]_{i j}$ can be renewed online by [34]:

$$
\left[T_{l}(L)\right]_{i j} \approx \frac{\operatorname{Fre}_{i j}^{l}(L-1)+\mu \cdot\left[\operatorname{flag}_{i j}^{l}(L)-\operatorname{Fre}_{i j}^{l}(L-1)\right]}{\operatorname{Fre}_{o i}^{l}(L-1)+\mu \cdot\left[\operatorname{flag}_{o i}^{l}(L)-\operatorname{Fre}_{o i}^{l}(L-1)\right]}, i, j \in\{1, \ldots, s\}, l \in\left\{1, \ldots, \mathrm{H}_{\mathrm{p}}\right\} .
$$

Through (8), the MC predictor can converge to the recent driving changes by stepwise updating its transition probabilities using the incrementally obtained driving information.

\subsubsection{Speed Forecasting Using Self-Learning Enhanced Markov Chain}

Benefiting from the online TPM updating technique, a novel speed forecasting method is proposed, whose three working phases are detailed as below.

(1) Parameter initializing phase. Before online TPM estimation, the size of Markov state space $\boldsymbol{s}$, the forgetting coefficient $\boldsymbol{\mu}$ and the preview length $\mathrm{H}_{\mathbf{p}}$ are specified. Afterwards, the MC state space $X_{\boldsymbol{a}}$ and the initial TPM group $\mathrm{T}_{\mathbf{i n i}}=\left\{T_{\mathbf{1}}(0), \ldots, T_{\mathbf{H}_{\mathbf{p}}}(0)\right\}$ are built. Note the $l$-th element in $\mathrm{T}_{\mathrm{ini}}$ is an s-by-s matrix, with all elements equaling to $1 / s$, and $s$ is set to 40 in this study.

(2) TPM updating phase. Sample the most recent acceleration states: $a(\mathrm{~L})=a_{j}$ and $a(\mathrm{~L}-l)=a_{i_{l}}$, where $a_{j}, a_{i_{l}} \in X_{a}$. Calculate $\operatorname{flag}_{i j}^{l}(L)$ and $\mathbf{f l a g}_{o i}^{l}(L)$ based on the state transition incidents from $a_{i_{l}}$ to $a_{j}, l=1, \ldots, \mathrm{H}_{\mathbf{p}}$. Then, the $L$-th transition frequency $\mathbf{F r e}_{i j}^{l}(L), \mathbf{F r e}_{\boldsymbol{o} i}^{l}(L)$ can be computed using the $(L-1)$ - $t h$ transition frequency $\mathbf{F r e}_{i j}^{l}(L-1), \mathbf{F r e}_{\boldsymbol{i}}^{l}(L-1)$ as indicated by $(7 \mathrm{~d})$ and (7e). Afterwards, each element within the $i_{l}$-th row of the $l$-step $\operatorname{TPM}_{l}(L)$ is renewed by (8), thus leading to the evolution of $\mathrm{T}_{\mathrm{G}}(\mathrm{L})=\left\{T_{1}(\mathrm{~L}), \ldots, T_{\mathrm{H}_{\mathrm{p}}}(\mathrm{L})\right\}$. Specially, if there is not enough historical driving data for TPM estimation $\left(L \leq H_{p}\right)$, initial TPM group $\mathrm{T}_{\mathrm{ini}}$ is adopted for velocity prediction.

(3) Prediction and post-processing phase. Given the updated TPM group $T_{G}(L)$ and the $L^{t h}$ acceleration state $a(\mathrm{~L})=a_{j}$, the acceleration in next $l$ steps is obtained by the probability-weighted 
average (expected value) of every interval middle point: $a^{*}(\mathrm{~L}+\boldsymbol{l})=\sum_{\mathrm{j}=1}^{\mathrm{s}}\left[T_{l}(\mathrm{~L})\right]_{i j} \cdot a_{j}$, if $a(\mathrm{~L}) \in I_{i}$.

265 Therefore, the $l$-step ahead velocity can be predicted by: $v^{*}(\mathrm{~L}+l)=v(\mathrm{~L})+\sum_{\mathrm{q}=1}^{\mathrm{q}=l} a^{*}(\mathrm{~L}+\mathrm{q}) \cdot \Delta \mathrm{T}$. Finally, to guarantee the smoothness of the forecasted speed profiles, the polynomial fitting algorithm is adopted for post-processing the velocity-forecast profiles. The sampling period $\Delta \mathrm{T}=1 \mathrm{~s}$.

To sum up, without using offline driving database, the enhanced Markov predictor is established and updated online based on the real-time measured driving data, where its working principle at L-th time step is depicted in Fig. 3.

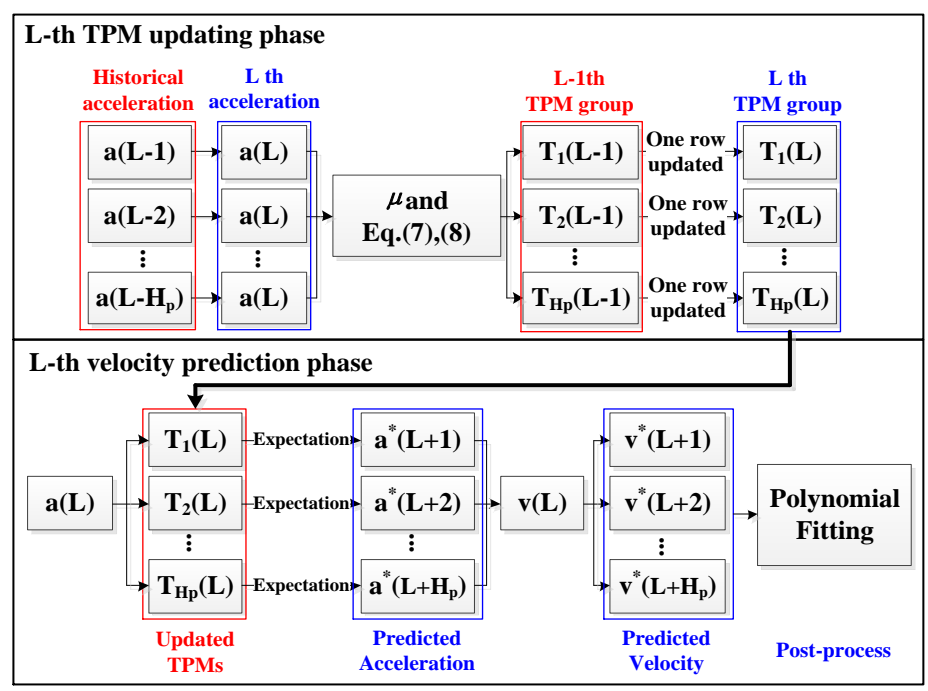

Fig. 3. Working flow within $\mathrm{L}^{\text {th }}$ updating and prediction phase.

\subsection{Battery SoC reference planning approach}

The plug-in property permits the vehicular battery to be recharged through the external grid power, which, hence, enables a way towards better fuel economy by consuming the low-cost electricity energy. Specifically, to realize the efficient utilization of battery energy under sophisticated traffic conditions, an explicit SoC reference trajectory is necessary for the MPC controller to track. Through narrowing the discrepancy between the real SoC and the reference one, the battery output behaviors can be properly regulated for adapting to different power requests.

Actually, depleting battery energy at various rates under multiple driving patterns may enhance the EMS control performance. Specifically, high-average power requests occur under highway driving conditions. In this case, the low-cost battery energy should be primarily utilized to save the hydrogen consumption, 
which leads to a high declining rate of SoC. In contrast, the low-average power requests in urban regions imply a relatively low SoC declining rate. Therefore, an adaptive $\mathrm{SoC}$ reference planning approach is designed for adjusting the battery SoC declining rates under multiple driving patterns, where Fig. 4 details the regulation mechanism of the proposed method.

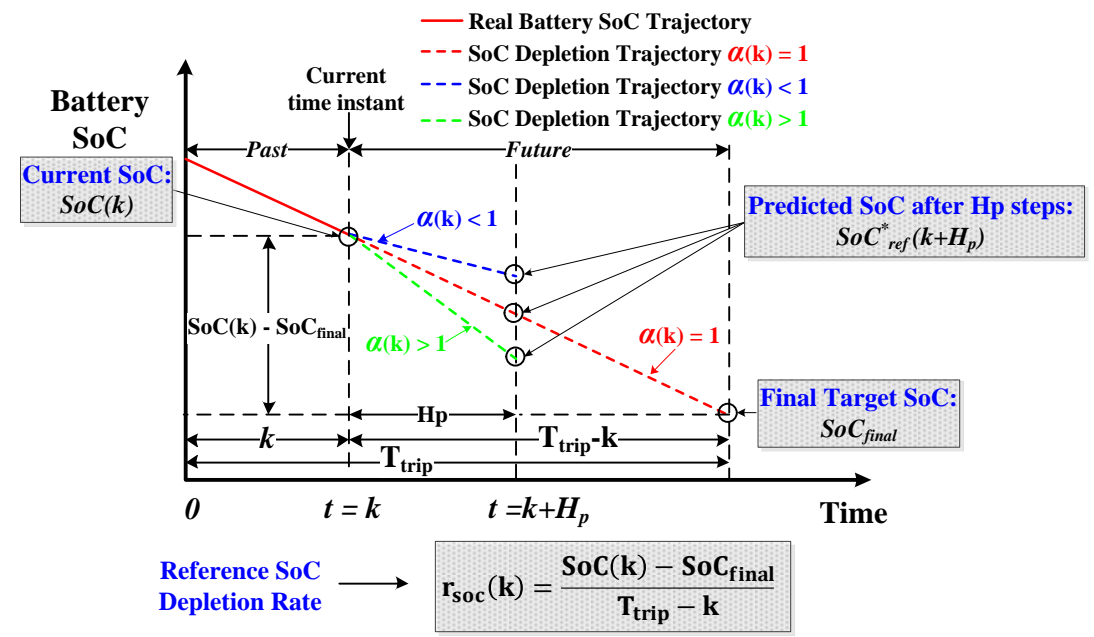

Fig. 4. Schematic diagram of the adaptive SoC reference generator.

The maturation of modern telematics techniques makes it possible to acquire the estimated trip duration information $\mathrm{T}_{\text {trip }}$ in advance. At $t=k$, let $\mathrm{SoC}(\mathrm{k})$ denotes the actual $\mathrm{SoC}, \mathrm{SoC}_{\text {final }}$ the terminal $\mathrm{SoC}$ target and $V_{\boldsymbol{k}}^{*}=\left[\mathrm{v}^{*}(\mathrm{k}+1), \ldots, \mathrm{v}^{*}\left(\mathrm{k}+\mathrm{H}_{\mathrm{p}}\right)\right]$ the forecasted speed profile, the predicted reference $\mathrm{SoC}$ at $\mathrm{t}=\mathrm{k}$

$$
S o C_{r e f}^{*}\left(k+H_{p}\right)=S o C(k)-\overbrace{\underbrace{\left(\frac{k_{\alpha}}{\left.1+\frac{v_{\text {std }}(k)}{v_{\text {ave }}(k)}\right)}\right)}_{\alpha(k)} \cdot \underbrace{\left(\frac{S o C(k)-S o C_{\text {final }}}{T_{\text {trip }}-k}\right)}_{r_{\text {soc }}(k)}}^{r_{\text {Soc }}^{\prime}(k)} \cdot H_{p}
$$

Where $r_{\text {soc }}$ represents the $\mathrm{k}$-th reference SoC declining rate concerning the remaining time of trip. Moreover, $\boldsymbol{\alpha} \in\left(0, k_{\alpha}\right]$ is the adjustment coefficient, where the constant parameter $k_{\alpha}>0$ specifies the upper boundary of $\alpha$. Besides, $r_{s o c}^{\prime}$ represents the modified SoC declining rate. Specifically, reducing $k_{\alpha}$ would slow down the overall SoC declining rate and thus may fail to entirely exploit the battery energy, whereas an exceeding large $k_{\alpha}$ would extremely accelerate the battery energy depletion, thus 
prolonging the vehicle's CS working period. Hence, a trade-off on the EMS performance against the battery energy utilization ratio should be made by using an appropriate $k_{\alpha}$.

Additionally, $v_{s t d}(k)$ and $v_{\text {ave }}(k)$ represent the standard deviation and mean value of the predicted velocity $V_{k}^{*}$, respectively. Note the forecasted speed trace $V_{k}^{*}$ with higher $v_{\text {ave }}$ and lower $v_{s t d}$ implies the highway scenario, leading to a larger $\alpha$. In contrast, a speed profile with lower $v_{\text {ave }}$ and higher $v_{\text {std }}$ indicates the urban scenario, meaning a smaller $\alpha$. Consequently, through the obtained $\alpha$ in different driving scenarios, the actual SoC declining rate $r_{S o c}^{\prime}$ is tuned by the following mechanism. If $\alpha>1, r_{s o c}^{\prime}$ is larger than the reference declining rate ( $\left.r_{s o c}\right)$. If $\alpha<1, r_{s o c}^{\prime}$ is smaller than $r_{s o c}$. Besides, $\alpha=0$ if and only if $v_{\text {ave }}=0$.

Furthermore, a linear SoC reference model (10) from literature [14] is introduced for comparison.

$$
\operatorname{SoC}_{r e f}^{*}(\mathrm{k})=S o C_{i n i}-\frac{k}{\mathrm{~T}_{\text {trip }}}\left(S o C_{\text {ini }}-\operatorname{SoC}_{\text {final }}\right)
$$

where $\mathrm{SoC}_{\mathrm{ini}}$ is the initial battery charge state. To ensure the battery operation safety, $\mathrm{SoC}_{\mathrm{ref}}^{*}$ is bounded within $\left[S o C_{\min }, S o C_{\max }\right]$, where $S o C_{\min }=0.3$ and $S o C_{\max }=0.9$. It should be mentioned that such range only specifies the boundary of SoC reference. When the actual $\mathrm{SoC}$ is beyond $[0.3,0.9]$, the "SoC emergency" working mode is triggered for urging SoC return to this range.

\subsection{Power Allocation Strategy using Model Predictive Control}

As the decision-maker with the PEMS framework, MPC acquires the optimal control sequences through minimizing the objective function at each time step. In this subsection, the MPC design process is presented in detail.

\subsubsection{Control-Oriented Model}

Let the symbol $\boldsymbol{x} \in R^{2 \times 1}$ denotes state variable, $\boldsymbol{u} \in R^{1 \times 1}$ the control input, $\boldsymbol{y} \in R^{1 \times 1}$ the system output, $\boldsymbol{w} \in R^{1 \times 1}$ the disturbance and $\boldsymbol{r} \in R^{2 \times 1}$ the reference trajectory, the control-oriented model is formulated as a linear discrete-time system (with 1s sampling period) as denoted by (11), where the MPC control horizon is identical to its prediction horizon $\left(H_{p}\right)$. 
$\boldsymbol{x}(\mathrm{k}+1)=\mathrm{A}(\mathrm{k}) \boldsymbol{x}(\mathrm{k})+\mathrm{B}(\mathrm{k}) \boldsymbol{u}(\mathrm{k})+\mathrm{C}(\mathrm{k}) \boldsymbol{w}(\mathrm{k})$

323

$$
\begin{aligned}
& \boldsymbol{y}(\mathrm{k})=\mathrm{D} \boldsymbol{x}(\mathrm{k})+\mathrm{E} \boldsymbol{u}(\mathrm{k})+\mathrm{G} \boldsymbol{w}(\mathrm{k}) \\
& \text { with }\left\{\begin{array}{l}
\boldsymbol{x}(\mathrm{k})=\left[\mathrm{SoC}(\mathrm{k}), \mathrm{P}_{\mathrm{fc}}(\mathrm{k}-1)\right]^{\mathrm{T}} \\
\boldsymbol{u}(\mathrm{k})=\Delta \mathrm{P}_{\mathrm{fc}}(\mathrm{k})=\frac{\mathrm{P}_{\mathrm{fc}}(\mathrm{k})-\mathrm{P}_{\mathrm{fc}}(\mathrm{k}-1)}{\Delta \mathrm{T}} \\
\boldsymbol{y}(\mathrm{k})=\mathrm{P}_{\mathrm{b}}(\mathrm{k}) \\
\boldsymbol{w}(\mathrm{k})=\mathrm{P}_{\mathrm{d}}(\mathrm{k}) \\
\boldsymbol{r}(\mathrm{k})=\left[\mathrm{SoC}_{\mathrm{ref}}, \mathrm{P}_{\mathrm{ref}}\right]^{\mathrm{T}}
\end{array}\right.
\end{aligned}
$$

324 Combined (11) with the DC power balance relationship (12a) and the first-order differential 325 approximation of SoC dynamics (12b), the system matrices $A, B, C, D, E, G$ are specified as (13).

$$
\left\{\begin{array}{l}
\mathrm{P}_{\mathrm{d}}(\mathrm{k})=\mathrm{P}_{\mathrm{b}}(\mathrm{k})+\eta_{\mathrm{DC} / \mathrm{DC}} \cdot \mathrm{P}_{\mathrm{fc}}(\mathrm{k}) \\
\frac{\operatorname{SoC}(\mathrm{k}+1)-\operatorname{SoC}(\mathrm{k})}{\Delta \mathrm{T}}=-\frac{\eta_{b}}{\mathrm{U}_{\mathrm{dc}}(\mathrm{k}) \cdot \mathrm{Q}_{\mathrm{b}}} \mathrm{P}_{\mathrm{b}}(\mathrm{k})
\end{array}\right.
$$

$$
\begin{aligned}
& \mathbf{A}(\mathrm{k})=\left[\begin{array}{cc}
1 & \frac{\Delta \mathrm{T} \cdot \eta_{D C / D C} \cdot \eta_{b}}{\mathrm{U}_{\mathrm{dc}}(\mathrm{k}) \cdot \mathrm{Q}_{\mathbf{b}}} \\
0 & 1
\end{array}\right] \quad \mathbf{B}(\mathrm{k})=\left[\begin{array}{cc}
\frac{\Delta \mathrm{T} \cdot \eta_{D C / D C} \cdot \eta_{b}}{\mathrm{U}_{\mathbf{d c}}(\mathrm{k}) \cdot \mathrm{Q}_{\mathbf{b}}} & 1
\end{array}\right]^{\mathrm{T}} \quad \mathbf{C}(\mathrm{k})=\left[\begin{array}{cc}
-\frac{\Delta \mathrm{T} \cdot \eta_{D C / D C} \cdot \eta_{b}}{\mathrm{U}_{\mathbf{d c}}(\mathrm{k}) \cdot \mathrm{Q}_{\mathbf{b}}} & 0
\end{array}\right]^{\mathrm{T}} \\
& \mathbf{D}=\left[\begin{array}{cc}
0 & -\eta_{D C / D C}
\end{array}\right] \quad \mathbf{E}=\left[-\eta_{D C / D C} \cdot \Delta \mathrm{T}\right] \quad \mathbf{G}=[1]
\end{aligned}
$$

\subsubsection{Multi-criteria performance index formulation}

329

Three metrics are taken into account of the MPC performance index, namely (i) FCS working efficiency,

(ii) FCS durability and (iii) SoC reference tracking ability. Consequently, the k-th control decision

$\mathrm{U}^{*}(\mathrm{k})=\left[\mathrm{u}^{*}{ }_{1}(\mathrm{k}), \ldots, \mathrm{u}_{\mathbf{H}_{\mathrm{p}}}^{*}(\mathrm{k})\right]$ is obtained by minimizing the multi-criteria cost function (14) subject to

$\operatorname{Obj}(\mathrm{k})=\sum_{i=1}^{\mathrm{H}_{\mathrm{p}}}[\boldsymbol{\pi}_{1} \cdot \underbrace{\left(\frac{\mathrm{P}_{\mathrm{fc}}(\mathrm{k}+\mathrm{i}-1)-\mathrm{P}_{\mathrm{ref}}}{\mathrm{P}_{\mathrm{fc}}^{\max }}\right)^{2}}_{L_{l}}+\boldsymbol{\pi}_{2} \cdot \underbrace{\left(\frac{\Delta \mathrm{P}_{\mathrm{fc}}(\mathrm{k}+\mathrm{i}-1)}{\Delta \mathrm{P}_{\mathrm{fc}}^{\max }}\right)^{2}}_{L_{2}}]+\pi_{3} \cdot \underbrace{\left(\frac{\mathrm{SoC}\left(\mathrm{k}+\mathrm{H}_{\mathrm{p}}\right)-\mathrm{SoC}_{\mathrm{ref}}}{\mathrm{SoC}_{\max }-\mathrm{SoC}_{\min }}\right)^{2}}_{L_{3}}$

$$
\left\{\begin{array}{l}
\mathrm{SoC}^{\mathbf{L}} \leq[\boldsymbol{x}]_{1} \leq \mathrm{SoC}^{\mathrm{H}} \\
\mathrm{P}_{\mathbf{f c}}^{\mathbf{L}} \leq[\boldsymbol{x}]_{2} \leq \mathrm{P}_{\mathbf{f c}}^{\mathbf{H}}
\end{array}\right.
$$


Where $\mathrm{P}_{\mathrm{fc}}^{\max }=30 \mathrm{~kW}, \Delta \mathrm{P}_{\mathrm{fc}}^{\max }=1 \mathrm{~kW} / \mathrm{s}, S o C_{\min }=0.3$ and $S o C_{\max }=0.9$. Moreover, to achieve a balanced EMS performance among three cost terms $\left(\boldsymbol{L}_{1}, \boldsymbol{L}_{2}, \boldsymbol{L}_{3}\right)$, the penalty coefficients $\left(\boldsymbol{\pi}_{1}, \boldsymbol{\pi}_{2}, \boldsymbol{\pi}_{3}\right)$ are tuned by trials and errors, based on the DP-optimized EMS performance. More details regarding the parameter tuning process can be found in [17]. As a result, $\pi_{1}, \pi_{2}, \pi_{3}$ are set as 1,8 and 80000 , respectively. Besides, the major objectives of $\boldsymbol{L}_{1}, \boldsymbol{L}_{2}, \boldsymbol{L}_{3}$ are attached as below:

- To guarantee the overall fuel cell operation efficiency, $L_{1}$ penalizes the FCS operating points deviating from the predefined reference one (the most efficient point), namely $P_{r e f}=P_{\eta}^{\max }$.

- As indicated in [35], restricting fuel cell power varying rate is beneficial to improving the FCSs' durability. Consequently, $L_{2}$ lays a penalty on large $\Delta P_{f c}$ to retard the fuel cell degradation imposed by dynamic loading conditions.

- The function of $L_{3}$ is to shrink the deviation between the real SoC and the reference one given by (9), namely $S o C_{r e f}=S o C_{r e f}^{*}\left(k+H_{p}\right)$. By tracking the terminal reference value in each prediction horizon and ignoring the intermediate processes, MPC could better restrain the fuel cell power transients introduced by improper SoC reference values.

Furthermore, constraint (15a) defines a wider SoC variation range for real-time optimization, where

$\operatorname{SoC}^{\mathbf{L}}=0.25$ and SoC $^{\mathbf{H}}=0.95$. However, if SoC emergency incident $(\mathrm{SoC}>0.9$ or $\mathrm{SoC}<0.3)$ appears, $\pi_{1}$ and $\pi_{2}$ are set to zero to urge SoC back to $[0.3,0.9]$. Constraints $(15 \mathrm{~b})-(15 \mathrm{~d})$ denote the physical limitations on fuel cell and battery, where $P_{f c}^{L}=0 W, P_{f c}^{\mathbf{H}}=30 k W, \Delta P_{f c}^{\mathbf{H}}=-\Delta P_{f c}^{L}=1 k W / s, P_{b}^{L}=-25 k W$ and $\mathrm{P}_{\mathbf{b}}^{\mathbf{H}}=50 \mathrm{~kW}$. Besides, (15e) sets the estimated DC power demands as the disturbance, where $P_{d}^{*}$ is calculated according to the forecasted speed $V_{k}^{*}$ and Eq. (1)-(2). Finally, the original optimization problem, namely minimizing (14) while respecting constraints (15), could be converted into a quadratic programming $(\mathrm{QP})$ problem and resolved by the well-established interior-point algorithm through calling the MATLAB-embedded quadprog function [17].

\section{Simulation and Discussion}

A simulation study is conducted in section IV to validate the performance of the presented EMS. All the simulations are performed in the MATLAB/Simulink environment (version: R2016a), which is installed 
in a desktop PC with an Intel Core i7-7700 CPU @ $3.30 \mathrm{GHz}$ and a 64G RAM. The discrete sampling

362

363

364

365

366

367

368

369

370

371

372

373 time step is set to 1 second.

\subsection{Speed Forecast Performance Verification}

In subsection 4.1, the performance of self-learning Markov speed-forecast method is validated. The Root-Mean-Square-Error (RMSE) is picked as the evaluation metric of forecast accuracy [36].

\subsubsection{Influences on forgetting coefficient $\mu$}

A small $\mu$ would reduce the updating rate of MC predictive model, while a large $\mu$ would shorten the effective memory length $D_{\mu}=1 / \mu$, reducing the completeness and reliability of the MC model. To explore the impacts on prediction performance by different $\mu$, the self-learning MC predictor with multiple forgetting coefficient candidates is evaluated under the INRETS driving cycle [32], where the prediction performance is shown in Fig. 5.

Fig. 5(b) and (c) thoroughly demonstrate the forecast results when $\mathrm{H}_{\mathrm{p}}=5 \mathrm{~s}$, where the average RMSE under different $\mu$ are respectively $1.1946 \mathrm{~m} / \mathrm{s}(\mu=0.1), 0.9766 \mathrm{~m} / \mathrm{s}(\mu=0.01)$ and $0.9594 \mathrm{~m} / \mathrm{s}(\mu=$ 0.002). Specifically, when $\mu=0.1$, the forecasted speed profiles tend to diverge significantly from the actual one. When $\mu$ reduces from 0.1 to 0.002 , the prediction performance improves greatly, especially in the circled regions, since the corresponding enlarged $D_{\mu}$ (from 10 to 500) enables adequate measurements for TPM estimation, thus improving the forecast precision. However, if $\mu$ continues to decrease, the forecast precision would decrease to some extent, as shown in TABLE II. This is because the enlarged $D_{\mu}$ (from 500 to 10000) would include superfluous information that cannot represent recent driving conditions, thus reducing the forecast reliability. Meanwhile, when $\mathrm{H}_{\mathrm{p}}=10 \mathrm{~s}$, similar tendency would also be detected. Hence, as a compromise between the prediction performance and the online memory burden, $\mu$ is set as $0.002\left(D_{\mu}=500\right)$ to handle the changeable driving conditions. 


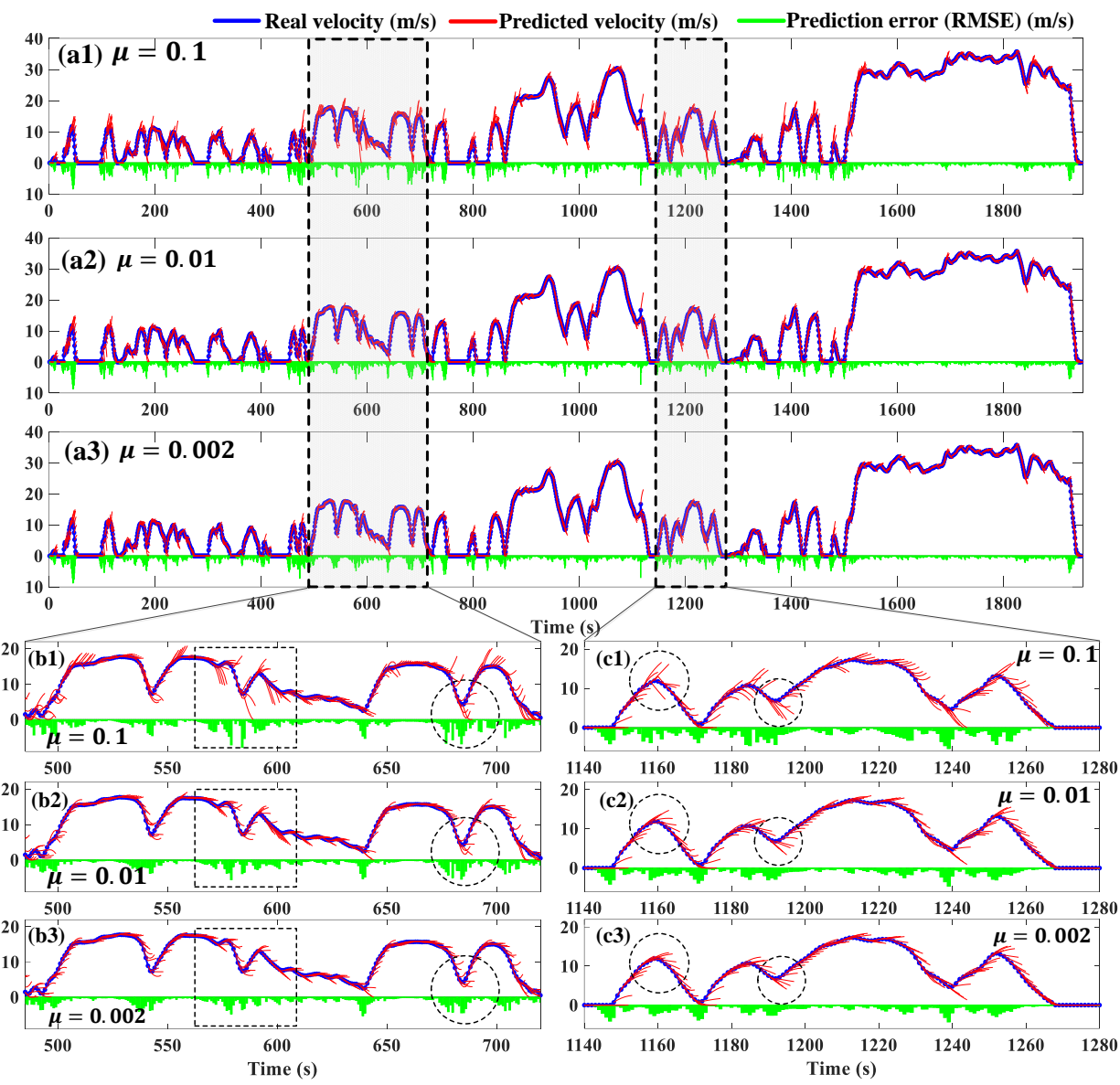

Fig. 5. Prediction performance $\left(\mathrm{H}_{\mathrm{p}}=5 \mathrm{~s}\right)$ with different $\mu$. (a) Velocity prediction results (global perspective). (b) Detail prediction results from 500s to 720 s. (c) Detail prediction results from 1140 s to 1280 s.

TABLE II. Average RMSE (m/s) with respect to different $D_{\mu}=1 / \mu$ under INRETS cycle

\begin{tabular}{|c|c|c|c|c|c|c|c|c|c|c|}
\hline $\boldsymbol{D}_{\boldsymbol{\mu}}$ & $\mathbf{5}$ & $\mathbf{1 0}$ & $\mathbf{5 0}$ & $\mathbf{1 0 0}$ & $\mathbf{2 0 0}$ & $\mathbf{5 0 0}$ & $\mathbf{1 0 0 0}$ & $\mathbf{2 0 0 0}$ & $\mathbf{5 0 0 0}$ & $\mathbf{1 0 0 0 0}$ \\
\hline $\boldsymbol{H}_{\boldsymbol{p}}=\mathbf{5 s}$ & 1.1946 & 1.1336 & 1.0102 & 0.9766 & 0.9624 & 0.9594 & 0.9713 & 0.9782 & 0.9828 & 0.9844 \\
\hline $\boldsymbol{H}_{\boldsymbol{p}}=\mathbf{1 0}$ & 2.5048 & 2.3823 & 2.1211 & 2.0513 & 2.0275 & 2.0198 & 2.0433 & 2.0550 & 2.0643 & 2.0661 \\
\hline
\end{tabular}

\subsubsection{Prediction Performance Comparison with Benchmark Predictors}

To compare the proposed method with benchmark approaches, two commonly used velocity-forecast methods, namely a multi-step MC (MSMC) and a BPNN predictor, are introduced as evaluation basis.

\section{- Benchmark Predictors Description}

Unlike the self-learning MC, the TPMs of MSMC predictor are estimated by (6) using offline stationary driving database. Additionally, as suggested in [36], a three-layer BPNN with 10 input neurons and 20 hidden neurons is adopted for speed forecasting.

\section{- Database Preparation}


The performances of benchmark predictors are highly dependent on the offline driving database. To cover vehicle's daily driving scenarios, several standard cycles with different driving patterns (urban/suburban/highway) are concatenated to form the offline driving database, as shown in Fig. 6(a). All standard cycles are extracted from ADVISOR. Note this database is used for the estimation of MC TPM and the training of BPNN, where $85 \%$ of data is for BPNN training while the remaining portion is for NN validation.

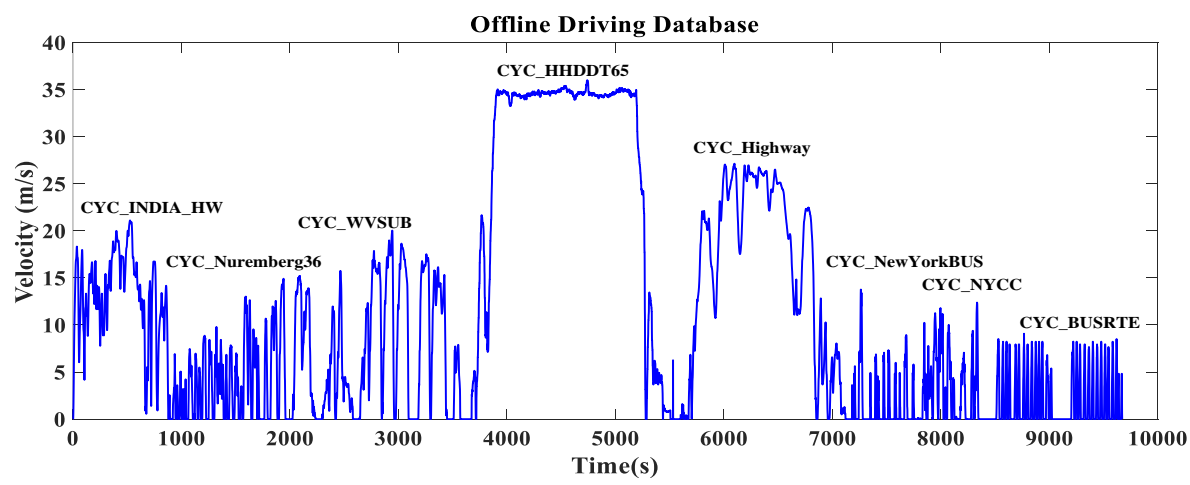

Fig. 6(a). Offline driving database for NN training and TPM estimation.

\section{- Performance Comparison Under Repetitive Driving Conditions}

Firstly, the performance of three predictors is compared under the Manhattan driving cycle, which represents the typical urban driving scenarios with very low average speed, frequent start-and-stops, and repetitive driving patterns.

Taken $H_{p}=5 s$ as an example, the prediction performance discrepancy is presented in Fig. 6(b)-(d). Specifically, both MSMC and BPNN predictor perform stably over the whole cycle. In comparison, due to the use of initial TPM groups, the online-learning Markov predictor results in the largest error in the first 200 seconds (Fig. 6(b1), (c1) and (d1)). As the updating of TPM group, its forecast errors gradually decrease to a lower level. Especially, as shown in the circled regions in Fig. 6(b2), (c2) and (d2), it performs even slightly better compared to benchmark predictors.

Moreover, Fig. 6(e) exhibits the error evolution processes (per 100s) of three predictors. Within the first 200s, the self-learning MC predictor leads to the significantly larger error compared to other predictors. Afterwards, due to the online TPM updating, its performance discrepancy against other predictors is shrinking. Specifically, it outperforms the MSMC predictor after 200s. After 500s, it even slightly outperforms the BPNN predictor until the trip end. 

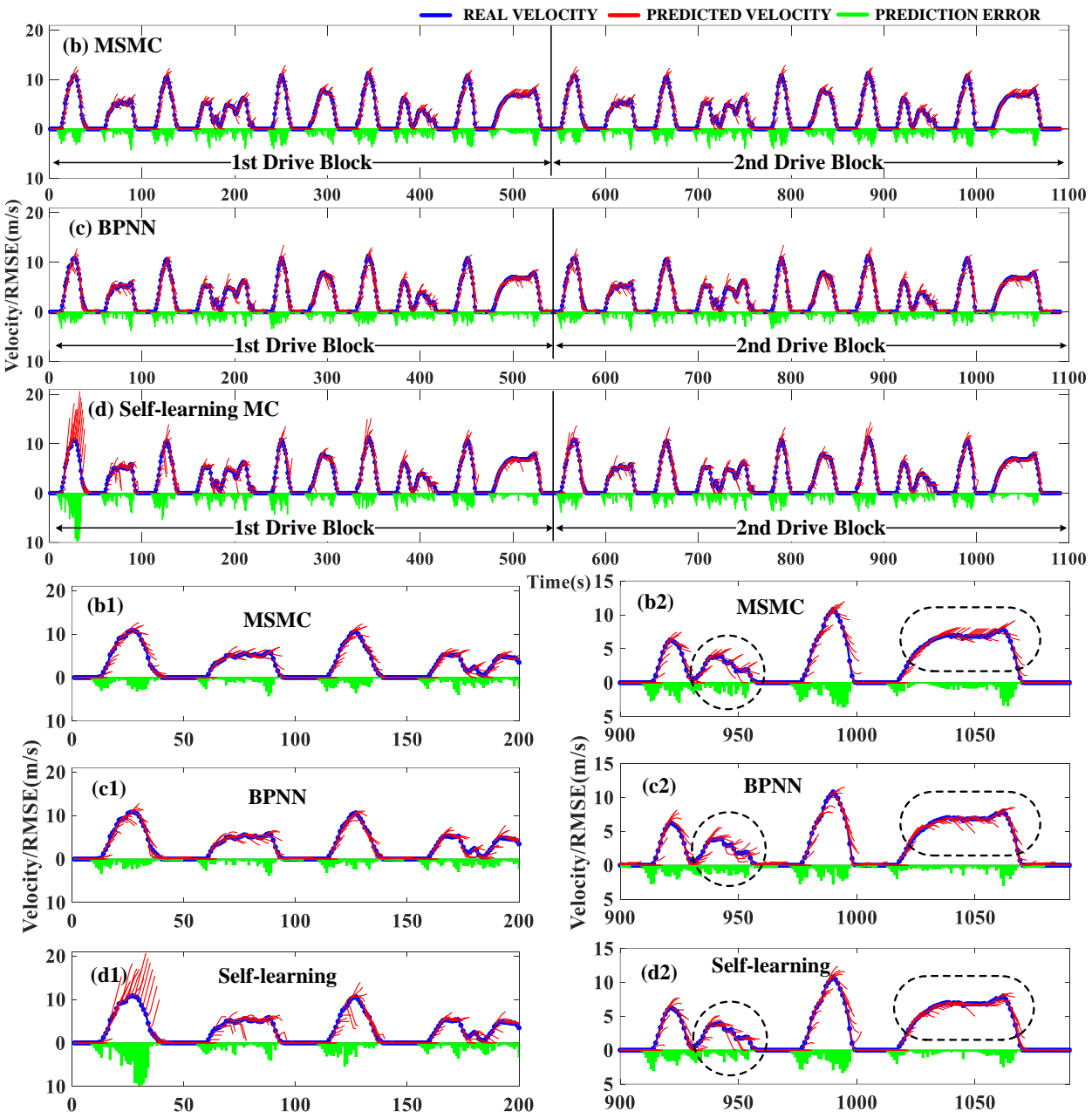

Fig. 6(b)-(d). Detail speed forecasting performance under Manhattan driving cycle $\left(H_{p}=5 s\right)$.

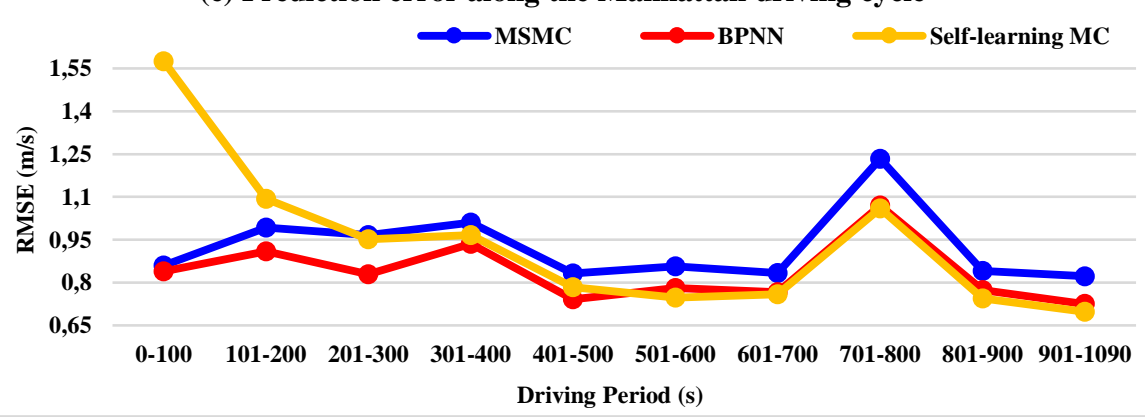

Fig. 6(e). Average RMSE comparison (per 100s) under Manhattan Driving Cycle.

422 Besides, the average RMSE along the trip is summarized in TABLE III. Unlike benchmark predictors,

423 under two identical drive blocks, the average RMSE for the proposed method is reduced by $20.4 \%$ (from

$424 \quad 1.0247 \mathrm{~m} / \mathrm{s}$ to $0.8156 \mathrm{~m} / \mathrm{s})$. This indicates the proposed method can acquire predictive knowledge from

425 the incrementally measured driving data and thus its dependency on offline driving database is reduced 
compared to benchmark predictors. Moreover, the effectiveness in enhancing the forecast precision by

427 the online-learning technique is also verified.

428

TABLE III. Average RMSE (m/s) under Manhattan driving cycle.

\begin{tabular}{|c|c|c|c|}
\hline & 1st Drive Block & 2nd Drive Block & Total \\
\hline MSMC & 0.9124 & 0.9208 & 0.9166 \\
\hline BPNN & 0.8279 & 0.8279 & 0.8279 \\
\hline Self-learning MC & 1.0247 & 0.8156 & 0.9206 \\
\hline
\end{tabular}

\section{- Performance Comparison Under Complex Driving Conditions}

To further evaluate the prediction performance under complex driving conditions, three standard cycles

10s to clearly show their performance discrepancies.
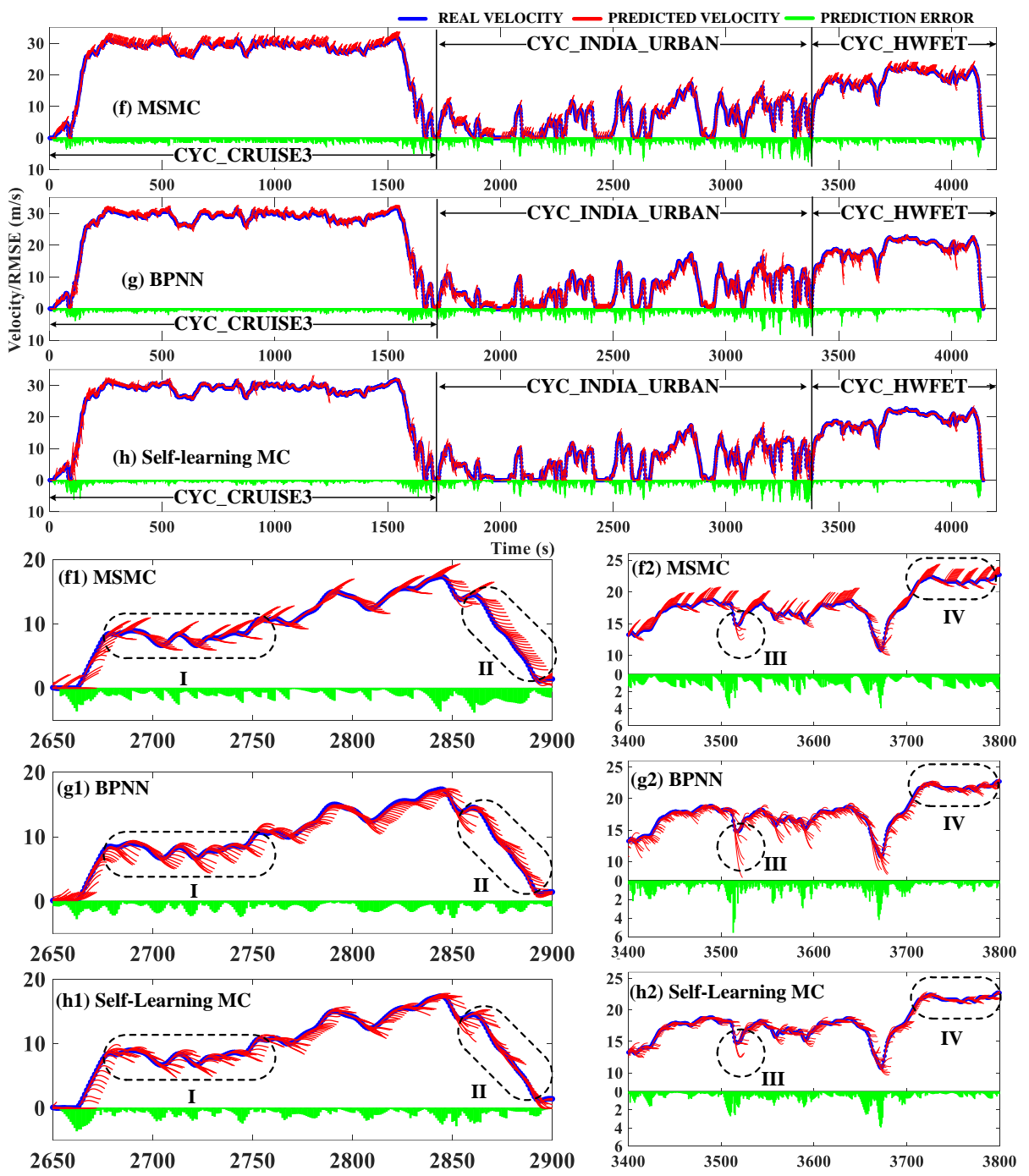

Fig. 6(f)-(h). Detail speed forecasting performance under multi-pattern driving cycle $\left(\mathrm{H}_{\mathrm{p}}=10 \mathrm{~s}\right)$. 
As can be seen, three predictors tend to generate smaller errors over the CRUISE3 and HWFET cycles, whereas larger errors appear over the INDIA_URBAN cycle. This is because the actual speed profile changes more sharply under city driving conditions, making higher forecast accuracy hard to achieve. Moreover, as depicted in the circled region I in Fig. 6(f1)-(h1), the forecasted speed profiles by MSMC predictor tend to remain the same tendency (rising or falling) as the input driving states, while other predictors can more precisely describe the future velocity dynamics. In comparison with BPNN benchmark, the online-learning Markov predictor can more promptly re-converge to the real speed trace after each inflection point, thus increasing the prediction accuracy during this period. Similarly, as shown in the zoomed regions II to IV, the proposed method shows the higher forecast accuracy and robustness compared to benchmark predictors.

The reason for such performance discrepancies is given as follows. Benchmark predictors learn future velocity dynamics from the offline stationary database and thus their predictive behavior toward each driving pattern is pre-determined. Nevertheless, owing to the absence of online-update mechanism, it is hard for them to fully adapt to the novel driving characteristics, thus compromising the forecast performance. In contrast, the proposed method can adjust its predictive behaviors by using the real-time updated TPMs, thus leading to the improved performance.

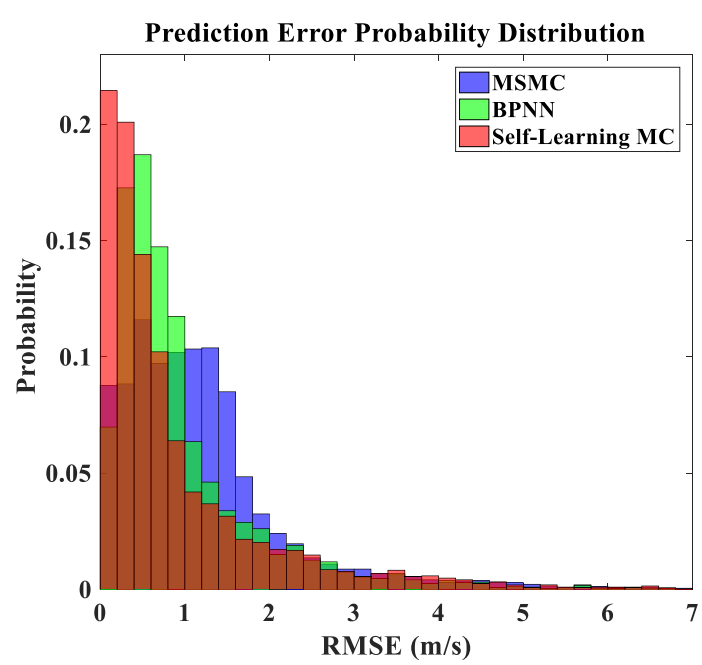
Fig. 6(i). Average RMSE probability distribution under multi-pattern testing cycle $\left(\mathrm{H}_{\mathrm{p}}=10 \mathrm{~s}\right)$. 
be confirmed that the proposed method can effectively characterize the future speed dynamics under changeable driving conditions with the reasonable forecast precision.

TABLE IV. Average RMSE (m/s) under combined driving cycle.

\begin{tabular}{|c|c|c|c|c|}
\hline & CYCLE_Cruise3 & CYCLE_INDIA_URBAN & CYCLE_HWFET & Total \\
\hline MSMC & 1.0365 & 1.4422 & 1.0540 & 1.2032 \\
\hline BPNN & 0.7577 & 1.3204 & 0.6839 & 0.9703 \\
\hline Self-learning MC & 0.6434 & 1.2662 & 0.6387 & 0.8936 \\
\hline
\end{tabular}

\subsection{Performance Verification of Energy Management Strategy}

The proposed predictive energy management strategy will be comprehensively evaluated in this subsection. In all case studies, the initial and terminal SoC values are set as 0.8 and 0.3 , respectively.

\subsubsection{Impacts on EMS performance by $k_{\alpha}, H_{p}$ and different $\mathrm{SoC}$ reference generators}

Several parameters of the proposed PEMS would heavily affect its performance, which should be carefully tuned before online implementations. This subsection presents a detailed analysis regarding the determination criteria of EMS parameters and the battery energy allocation performance comparison with linear SoC reference (10). Please note that the same multi-pattern driving cycle in Fig. 6(f) is used as the testing cycle, whose speed and power demand profiles are depicted in Fig. 7(a).

\section{- Determination of SoC reference adjusting factor boundary $\boldsymbol{k}_{\alpha}$}

As mentioned before, $k_{\alpha}$ controls the upper boundary of the adjusting factor $\alpha$. To find a proper $k_{\alpha}$ for online application, the MPC-based EMS with multiple $k_{\alpha}$ candidates (1 to 5) is tested under the multipattern driving cycle, where $H_{p}$ is set as 5 seconds. Fig. 7(b) displays the obtained SoC traces. Apparently, if $k_{\alpha}=1$, larger final $\mathrm{SoC}$ value is detected compared to other $k_{\alpha}$ settings, meaning the overall SoC declining rate is not enough to ensure the full utilization of battery energy. In contrast, although using larger $k_{\alpha}$ can ensure a deeper battery discharge, if $k_{\alpha}>2$, the overlarge SoC declining rates would contribute to the $\mathrm{SoC}$ emergency events ( $\mathrm{SoC}<0.3$, as shown in the zoomed area). Hence, set $k_{\alpha}$ as two is a trade-off decision between the battery working safety and the exploitation rate of battery energy. 


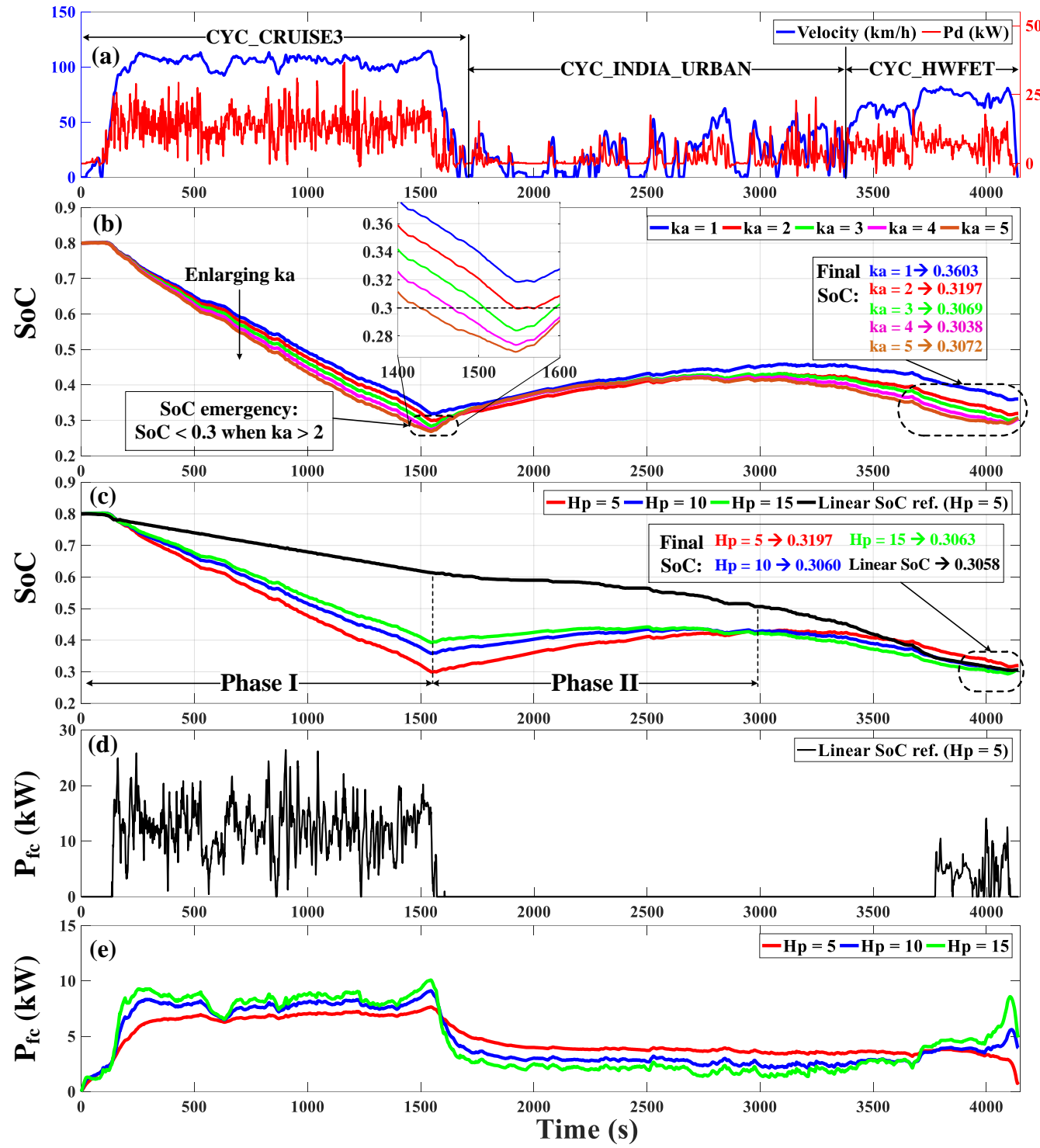

Fig. 7. EMS performance comparison under different parameter settings. (a) Velocity and power request profile of the multipattern driving cycle. (b) SoC profiles under multiple $k_{\alpha}\left(\mathrm{H}_{\mathrm{p}}=5 \mathrm{~s}\right)$. (c) SoC regulation performance comparison by different reference generators and different $\mathrm{H}_{\mathrm{p}}\left(k_{\alpha}=2\right)$. (d) Fuel cell power profile using linear SoC reference $\left(\mathrm{H}_{\mathrm{p}}=5 s\right)$. (e) Fuel cell power profiles using the proposed $\mathrm{SoC}$ reference and different $\mathrm{H}_{\mathrm{p}}$.

\section{- EMS performance discrepancy using different $\mathrm{SoC}$ reference generators}

Given $k_{\alpha}=2$, the performances of MPC-based EMS with different SoC references are compared in Fig. 7(c) to Fig. 7(e). For the linear SoC reference based EMS, Fig. 7(c) and (d) only depict its performance when $\mathrm{H}_{\mathrm{p}}=5 \mathrm{~s}$, while the performance under other $\mathrm{H}_{\mathrm{p}}$ settings is given in TABLE V.

Fig. 7(c) depicts the SoC regulation performance by two types of reference generators. The linear reference model (10) (black curve) tends to evenly distribute battery energy over the entire trip. Due to the extremely low external power demand in phase II, despite the fuel cell has been turned off in this 
phase (Fig. 7(d)), the SoC declining rate is still slightly lower than that in phase I. In contrast, the adaptive SoC reference generator $\left(\mathrm{H}_{\mathrm{p}}=5 \mathrm{~s}\right.$, red curve) can effectively adjust battery energy usage under different driving patterns. Specifically, the battery energy is largely used due to the high average power demand in highway scenario (phase I), whereas the battery tends to be recharged or less used in urban scenario (phase II).

Guided by the linear SoC reference (10), the EMS adjusts the fuel cell output power in an aggressive way, as displayed in Fig. 7(d). Large power transients and frequent start-and-stop cycles can be observed over the testing cycle, especially from 200 s to 1500 s and from 3700 s to 4150 s. Such working conditions would accelerate the degradation of fuel cell system, leading to the compromised fuel cell durability. In contrast, as shown in Fig. 7(e), guided by the proposed SoC reference model (9), fuel cell works stably around the reference point, with few power transients. Besides, no FC on-off cycles can be observed within the entire testing cycle.

Moreover, TABLE V summarizes the EMS performance discrepancies under different SoC reference models, where $m_{\mathrm{H}_{2}}$ denotes the actual $\mathrm{H} 2$ mass consumption, $m_{\mathrm{H}_{2}, \text { equ }}$ the equivalent $\mathrm{H} 2$ consumption that converts the terminal $\mathrm{SoC}\left(\mathrm{SoC}_{\text {end }}\right.$ ) deviation from 0.3 into corresponding $\mathrm{H} 2$ consumption [31], $\left|\overline{\Delta P_{F C}}\right|$ the average fuel cell power changing rate and $T_{\text {step }}$ the online calculation time per step. It can be clearly seen that, after using the proposed SoC reference model (9), $m_{H_{2}, \text { equ }}$ and $\left|\overline{\Delta P_{F C}}\right|$ are greatly reduced compared to those of linear SoC reference based EMS. Besides, both SoC reference based EMSs perform similarly in terms of final SoC and online computation efficiency.

To sum up, the proposed SoC reference model (9) is capable of depleting battery energy in a flexible manner regarding different power requirements, thus enhancing the rationality of battery energy allocation in contrast to linear reference model (10). Furthermore, benefiting from such proper battery energy distribution, the EMS can greatly suppress the fuel cell power spikes and effectively improve the fuel cell working efficiency.

\section{- Determination of prediction horizon $\mathrm{H}_{\mathrm{p}}$}


$515 \mathrm{H}_{\mathrm{p}}$ defines the length of speed prediction and the size of real-time optimization problem, which would

516 have large impacts on both online computation efficiency and EMS performance. With different $\mathrm{H}_{\mathrm{p}}$

517 settings, the fuel cell power and SoC profiles of the adaptive SoC reference based EMS are illustrated

518 in Fig. 7(c) and (e), respectively, where the related quantitative results are listed in TABLE V. It is clear

519 that increasing $H_{p}$ would enlarge $m_{H_{2}, \text { equ }}$ but guarantee a deeper battery discharge. Moreover, $\left|\overline{\Delta P_{F C}}\right|$

520 and $T_{\text {step }}$ increase with the growth of $\mathrm{H}_{\mathrm{p}}$. Therefore, set $\mathrm{H}_{\mathrm{p}}$ as five is a trade-off decision among the

521 following metrics, namely the H2 consumption conservation, the fuel cell power transients and the

522 online calculation burden.

523

TABLE V. EMS Performance discrepancies under different $\mathrm{H}_{\mathrm{p}}$ and different types of SOC reference.

\begin{tabular}{|c|c|c|c|c|c|c|}
\hline SoC reference & $\mathbf{H}_{\mathbf{p}}$ & $\mathbf{m}_{\mathbf{H}_{\mathbf{2}}}(\mathbf{g})$ & $\mathbf{m}_{\mathbf{H}_{\mathbf{2}}, \mathbf{e q u}}(\mathbf{g})$ & $\mathbf{S o C}_{\text {end }}$ & $\left|\overline{\Delta \mathbf{P}_{\mathbf{F C}}}\right| \mathbf{( W / s )}$ & $\mathbf{T}_{\text {step }}(\mathbf{m s})$ \\
\hline Linear Eq. (10) & 5 & 256.0 & 253.9 & 0.3058 & 438.2 & 16.89 \\
\hline Linear Eq. (10) & 10 & 254.4 & 252.4 & 0.3057 & 343.1 & 23.79 \\
\hline Linear Eq. (10) & 15 & 253.8 & 251.8 & 0.3057 & 298.5 & 32.89 \\
\hline Adaptive Eq. (9) & 5 & 236.7 & 229.8 & 0.3197 & 7.3 & 17.48 \\
\hline Adaptive Eq. (9) & 10 & 234.0 & 231.8 & 0.3060 & 14.3 & 25.68 \\
\hline Adaptive Eq. (9) & 15 & 235.8 & 233.6 & 0.3063 & 21.8 & 36.73 \\
\hline
\end{tabular}

524

525

526

527

528

529

530

531

\subsubsection{Comparative Study against Benchmark Control Strategy}

To thoroughly evaluate the proposed PEMS, two commonly used control strategies are introduced as comparison basis, where the DP-based strategy is deemed as the upper benchmark and the CD-CS strategy is deemed as the lower benchmark.

\section{- Benchmark EMS Description}

As the upper benchmark, DP-based strategy aims at obtaining the global optima by minimizing the predefined cost function. In this study, the DP problem is denoted as below:

$$
\min _{\Delta P_{f c} \in \mu_{f c}} \sum_{k=0}^{N-1} \Delta m_{H_{2}}\left(\Delta P_{f c}(\mathrm{k})\right) \cdot \Delta T
$$

$$
\text { s.t. }\left\{\begin{array}{l}
0.3 \leq \operatorname{SoC}(k) \leq 0.9 \\
0 \leq P_{f c}(k) \leq 30 \mathrm{~kW} \\
-1 \mathrm{~kW} / \mathrm{s} \leq \Delta P_{f c}(k) \leq 1 \mathrm{~kW} / \mathrm{s} \\
-25 \mathrm{~kW} \leq P_{b}(k) \leq 50 \mathrm{~kW} \\
S o C_{0}=0.8, P_{f c_{0}}=0 \mathrm{~W} \\
\operatorname{SoC}_{N}=0.3
\end{array}\right.
$$


533 feasible region for $\Delta P_{f c}$, where the resolution of control (input) variable is set as $1 \mathrm{~W} / \mathrm{s}$. Besides, the preset level 0.3 .

In contrast, the CD-CS strategy controls the FC output power based on the SoC value. Specifically, when SoC is higher than the threshold 0.3, the FCS switches off. When SoC is lower than this threshold, the FCS switches on and the reference working point is set as $P_{f c_{\max }}=30 \mathrm{~kW}$. To guarantee the fairness for EMS comparison, the permissible FC power-changing rate for CD-CS strategy is bounded within $[-1,1] \mathrm{kW} / \mathrm{s}$, which is identical to DP-based and MPC-based EMS.

\section{- Evaluation against benchmark EMSs}

Three EMSs are performed under two multi-pattern testing cycles (namely CYCLE1 and CYCLE2).

Note $H_{p}$ is set to $5 \mathrm{~s}$ and $k_{\alpha}$ is set to 2 . The performance discrepancies among three EMSs are shown in close to the DP benchmarks, while the CD-CS strategy depletes the battery energy more quickly than other strategies. Specifically, due to the availability of entire trip information, DP strategy can urge the FCS working steadily along the trip with few power transients. In contrast, MPC-based EMS can greatly restrict the FC power transients. The CD-CS strategy switches the FCS off when the SoC is higher than 0.3. Afterwards, when the battery SoC drops below 0.3, the FCS frequently turns on and off to maintain the SoC level. As a result, much more FC power transients can be observed within the entire CS phases. TABLE VI summaries the numerical results of three strategies. In contrast to CD-CS benchmark, the MPC-based EMS can respectively reduce $m_{\mathrm{H}_{2}, \text { equ }}$ by $15.30 \%$ and $12.05 \%$ under both testing cycles. Moreover, compared to DP benchmark, its performance gaps on $m_{H_{2}, \text { equ }}$ are respectively $3.74 \%$ and 4.88\%. In addition, the MPC-based EMS can suppress the FC power transients under both testing cycles by $96.80 \%$ and $94.90 \%$ compared to CD-CS strategy, thus reducing the possibility of fuel cell performance degradation imposed by dynamic load shifts. Finally, it can be observed that as a global 
optima-searching approach, DP benchmark consumes the largest amount of computation time, while the

559 online computation burden for MPC-based EMS is adequately smaller in contrast to the sampling period

560 (1s) and thus is affordable for online implementations.
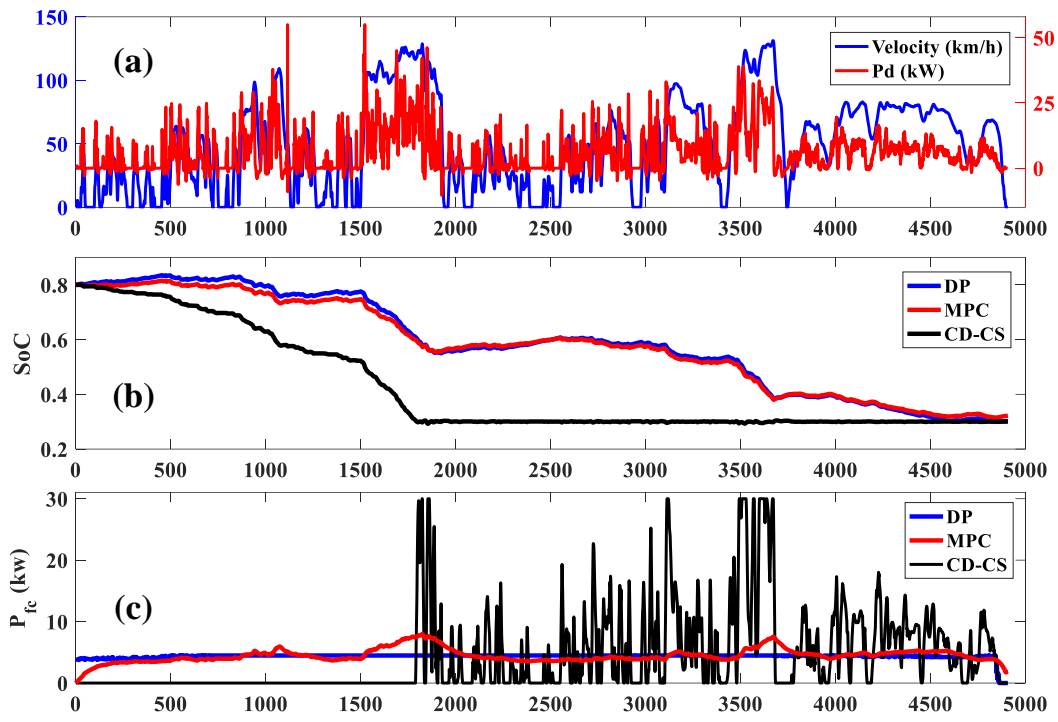

561

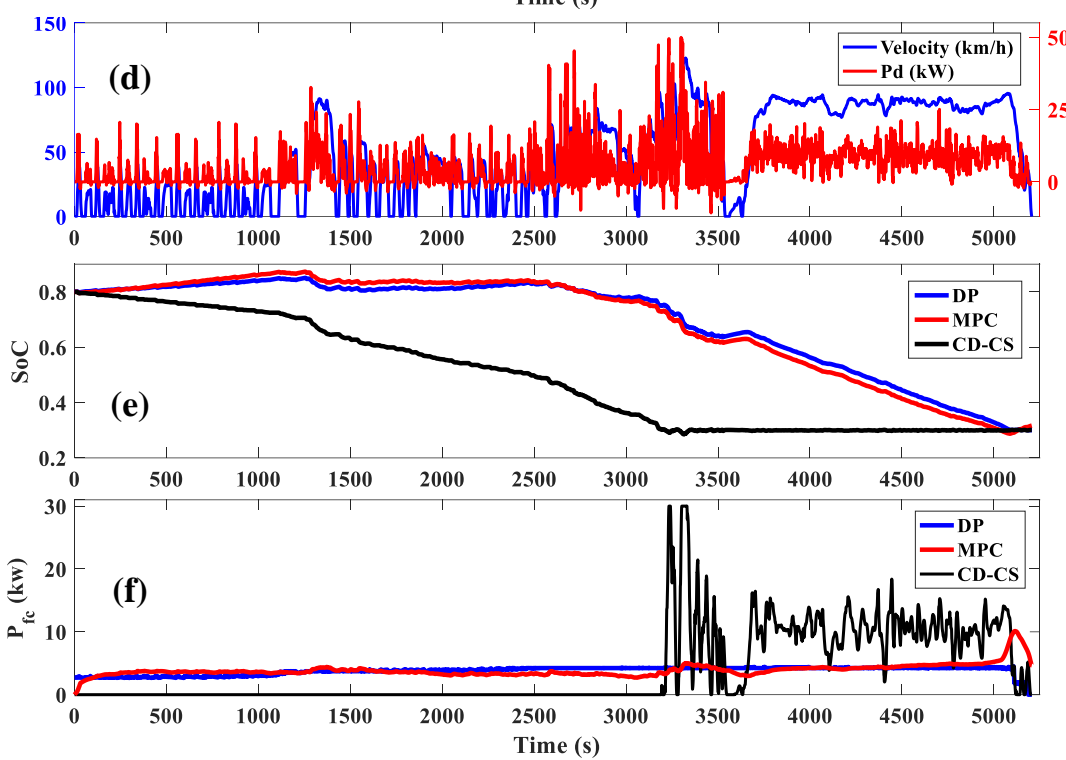

562

564

565

TABLE VI. EMS results compared to benchmark strategies.

\begin{tabular}{|c|c|c|c|c|c|c|c|}
\hline \multicolumn{2}{|c|}{ EMS } & $\mathbf{m}_{\mathbf{H}_{\mathbf{2}}}(\mathbf{g})$ & $\mathbf{m}_{\mathbf{H}_{2}, \text { equ }}(\mathbf{g})$ & $\mathbf{S o C}_{\text {end }}$ & $\left|\overline{\mathbf{A P}_{\mathbf{F C}}}\right|(\mathbf{W} / \mathbf{s})$ & $\mathbf{T}_{\text {total }}(\mathbf{s})$ & $\mathbf{T}_{\text {step }}(\mathbf{m s})$ \\
\hline \multirow{3}{*}{ CYCLE1 1} & DP & 245.9 & 245.9 & 0.3000 & 5.6 & 412.36 & N/A \\
\cline { 2 - 8 } & $\mathbf{M P C}$ & 262.8 & 255.1 & 0.3218 & 11.8 & 81.13 & 16.39 \\
\cline { 2 - 8 } & $\mathbf{C D}-\mathbf{C S}$ & 301.6 & 301.2 & 0.3011 & 375.1 & 11.48 & 2.32 \\
\hline \multirow{3}{*}{ CYCLE2 } & DP & 223.5 & 223.5 & 0.3000 & 7.3 & 489.56 & $\mathrm{~N} / \mathrm{A}$ \\
\cline { 2 - 8 } & $\mathbf{M P C}$ & 240.3 & 234.4 & 0.3168 & 9.4 & 87.72 & 17.20 \\
\cline { 2 - 8 } & $\mathbf{C D}-\mathbf{C S}$ & 266.8 & 266.5 & 0.3008 & 185.2 & 15.56 & 3.05 \\
\hline
\end{tabular}

Fig. 8 (a)-(f). Performance discrepancy of three control strategies under multi-pattern driving cycles. (a)-(c) Evaluation results on CYCLE1. (d)-(f) Evaluation results on CYCLE2.

Influences on EMS performance imposed by $T_{\text {trip }}$ estimation errors 
As indicated in (9), the planning of battery energy depletion is realized by assuming the information of estimated trip time can be obtained in advance. Nevertheless, many uncertain events, like the traffic congestions or the driving routes adjustment, will eventually lead to the discrepancy between the estimated $\mathbf{T}_{\text {trip }}$ and the actual one. To study the possible influences on EMS performance, different levels of trip duration errors (ranging from $-50 \%$ to $50 \%$ of the real trip time) are applied to the proposed SoC reference generator (9). Positive errors indicate the estimated trip duration is larger in contrast to the real trip time, whereas negative ones mean the opposite. Under $\pm 50 \%$ estimation errors, the MPC-based EMS is performed under CYCLE1 and CYCLE2, where the performance gaps against the CD-CS benchmark are given in Fig. 8(g)-(j).

\section{$>$ Fuel Economy Comparison}

As shown in Fig. 8(g), when positive errors (0 to 50\%) appear, the performance gap on the actual $\mathrm{H} 2$ consumption against the CD-CS benchmark is shrinking on both testing cycles. This is because the enlarged $\mathrm{T}_{\text {trip }}$ would slow down the SoC declining rate, resulting in the larger amount of remaining battery energy (see Fig. 8(h)). However, since the FCS's working efficiency can be maintained relatively stable, the performance on the equivalent $\mathrm{H} 2$ consumption remains almost the same as the "zero-error" conditions (Fig. 8(i)). In contrast, when negative errors occur (0\% to $-50 \%)$, the adaptive SoC reference generator would lead to a faster battery energy usage, thus extending the CS driving phases. Consequently, the FCS tends to work at higher power level for both supplying the external power demands and sustaining SoC level, thus compromising fuel efficiency performance.

\section{$>$ FC Power Transients Comparison}

Additionally, as depicted in Fig. 8(j), the FC power transients would be enlarged if negative errors appear, whereas it would remain nearly unchanged when positive errors occur. This is because the prolonged CS phases imposed by the minus errors enforce fuel cell operating in a more active manner, thus increasing the power spikes. In contrast, the period of CS working stage would be reduced (or even eliminated) under positive errors and thus the FC power transients would remain almost the same level as "zero-error" conditions. 
(g) Actual H2 Consumption Saving v.s. CD-CS Strategy

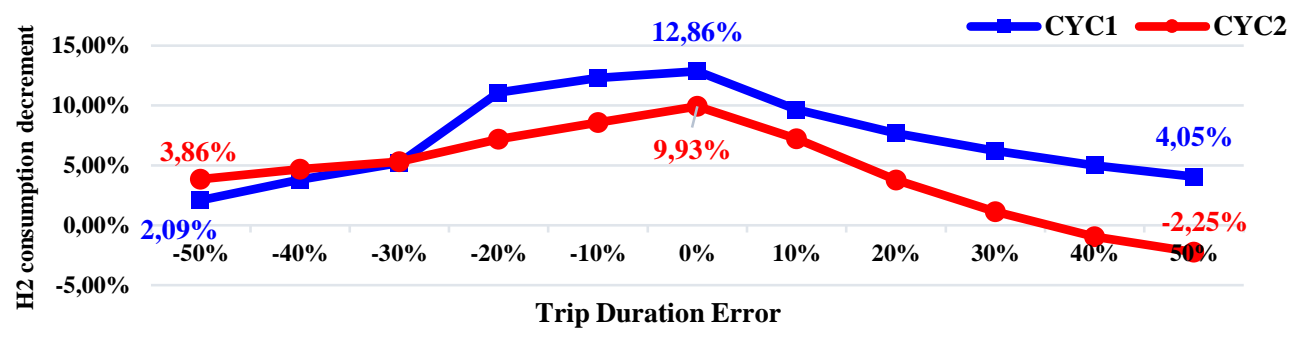

(h) Final SoC Increment v.s. CD-CS Strategy

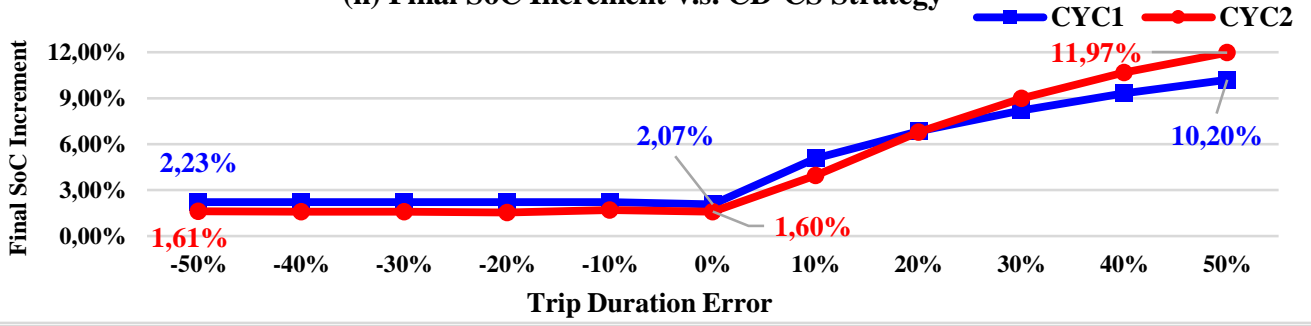

(i) Equivalent $\mathrm{H} 2$ Consumption Saving v.s. CD-CS Strategy

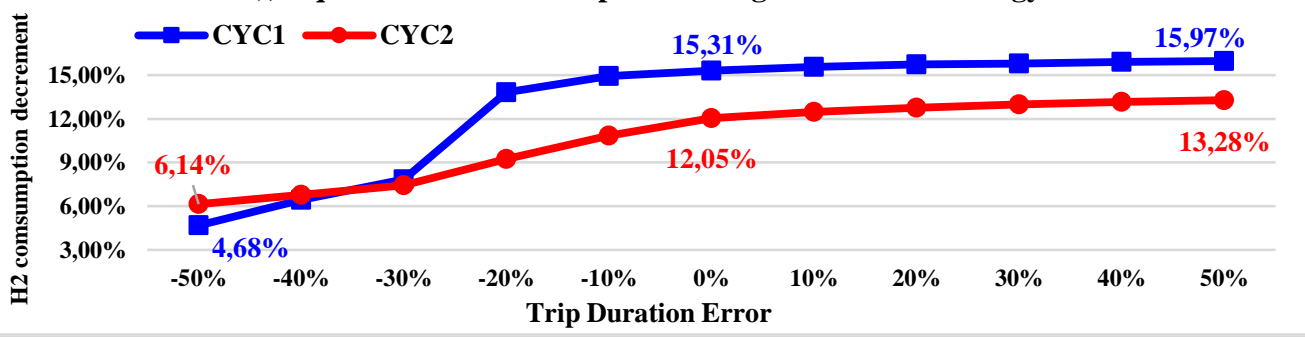

(j) FC Power Transients Decrement v.s. CD-CS Strategy

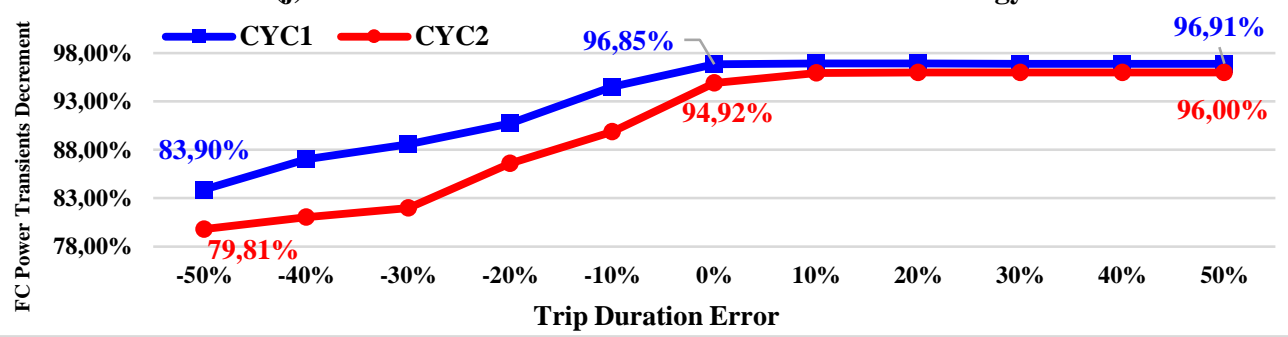

Fig. 8(g)-(j). MPC-based EMS performance deviations against CD-CS strategy under different trip duration errors.

598 Overall, despite $\pm 50 \%$ trip duration errors, the MPC-based EMS can effectively (1) improve the fuel 599 efficiency by at least 4.68\% (CYCLE1) and 6.14\% (CYCLE2), and (2) reduce the FC power spikes by at least $83.90 \%$ (CYCLE1) and $79.81 \%$ (CYCLE2), compared to CD-CS strategy. To sum up, in face of the trip the duration errors imposed by unpredictable traffic conditions, the proposed EMS could still outperform the CD-CS benchmark, thus denoting its potential for actual applications.

604 This work adopts an available powertrain sizing configuration from [16] for EMS development, where the FCS is functioned as a range extender. Under such powertrain configuration, battery can offer the 
majority of vehicular power demand if its $\mathrm{SoC}$ is high (e.g. $S o C_{i n i}=0.8$ in all case studies). In this case, the FCS does not necessarily work towards its nominal power level. Moreover, benefiting from the previewed trip knowledge, DP and MPC-based strategies can better anticipate and control the SoC drop compared to the CD-CS strategy, thus making as much FCS working points as possible towards the most efficient point $(\sim 5 \mathrm{~kW})$ for saving $\mathrm{H} 2$ and enhancing FCS working efficiency. Although the FCS seems to be oversized when battery $\mathrm{SoC}$ is high, it is still meaningful of using a $30 \mathrm{~kW}$ FCS, since it can provide sufficient traction power to ensure the vehicle's operation safety when SoC emergency event occurs. Furthermore, if we slightly downsize the battery capacity in the current powertrain, larger portion of vehicular power demand would be supplied by FCS. As a result, the corresponding increased average FCS power level would lead to a higher FCS efficiency, and escaping from extremely low loading conditions would contribute to the FCS lifetime extension.

\section{Conclusion}

This paper proposes a multi-criteria power allocation strategy for a fuel cell/battery-based plug-in hybrid electric vehicle. Firstly, a novel speed-forecast approach using online-learning Markov Chain is designed. Afterwards, a state-of-charge reference planning approach is designed for guiding battery energy allocation. Combining the speed-forecast results and the reference SoC value, MPC acquires the optimal control action through minimizing the multi-purpose objective function in a finite time horizon. The important findings in this research are detailed as below:

1) Compared to benchmark predictors, the benefits of the online-learning Markov velocity-forecast approach lie in: (a) the reduced dependency on the offline driving database since its TPMs are identified online using the recently measured data; (b) the higher prediction robustness towards the new driving conditions since its predictive behaviors can be adjusted by the real-time updated TPMs. Moreover, validation results show the proposed method is more capable of describing the future speed dynamics under complicated driving conditions.

2) Assisted by the estimated trip duration information and the speed-forecast results, the rapid SoC reference planning approach is able to adjust the battery energy-declining rate with respect to various 
632 driving patterns, thus enhancing the rationality in battery energy allocation in contrast to linear SoC 633 reference model.

634 3) In contrast to CD-CS benchmark, the proposed EMS can conserve the equivalent hydrogen 635 consumption by over $12.05 \%$ and suppress the average FC power transients by over $94.90 \%$, indicating 636 the enhanced FCS efficiency and durability. Furthermore, in spite of trip time prediction errors, the 637 presented control strategy is still able to bring performance improvement over CD-CS benchmark, 638 which should be deemed as the potential benefits for its real-world implementations.

639 Future works will focus on developing a data-driven SoC reference estimation approach, which would 640 be embedded into the EMS of a FCPHEV dedicated to postal-delivery.

\section{Acknowledgement}

642 This work has gained supports from the China Scholarship Council (CSC) and the EIPHI Graduate 643 School (contract "ANR-17-EURE-0002"). 
[1]. X. Lv, Y. Qu, Y. Wang, C. Qin, G. Liu. A comprehensive review on hybrid power system for PEMFC-HEV: Issues and strategies. Energy Conv. Manag., Vol. 171, Pages: 1273-1291, Sep. 2018.

[2]. H. Fathabadi. Fuel cell hybrid electric vehicle (FCHEV): Novel fuel cell/SC hybrid power generation system. Energy Conv. Manag., Vol. 156, Pages: 192-201, Jan. 2018.

[3]. M. Sorrentino, V. Cirillo, L. Nappi. Development of flexible procedures for co-optimizing design and control of fuel cell hybrid vehicles. Energy Conv. Manag., Vol. 185, Pages: 537-551, Apr. 2019.

[4]. Z. Hua, Z. Zheng, M.C. Péra, F. Gao, Remaining useful life prediction of PEMFC systems based on the multi-input echo state network, Appl. Energy, Vol. 265, 2020, 114791.

[5]. S. Overington, S. Rajakaruna. High-Efficiency Control of Internal Combustion Engines in Blended Charge Depletion/Charge Sustenance Strategies for Plug-In Hybrid Electric Vehicles. IEEE Trans. Veh. Technol., Vol. 64, Jan. 2015, Pages 48-61.

[6]. Y. Wu, A. Ravey, D. Chrenko, A. Miraoui, Demand side energy management of EV charging stations by approximate dynamic programming, Energy Conv. Manag., Vol. 196, 2019, Pages 878-890.

[7]. A. Ravey, B. Blunier, A. Miraoui. Control Strategies for Fuel-Cell-Based Hybrid Electric Vehicles: From Offline to Online and Experimental Results. IEEE Trans. Veh. Technol., Vol. 61, Jul. 2012.

[8]. Z. Chen, C. Mi, R. Xiong, J. Xu, C. You. Energy management of a power-split plug-in hybrid electric vehicle based on genetic algorithm and quadratic programming. J. Power Sources, Vol. 248, Feb. 2014, Pages 416-426.

[9]. R. Fernández, S. Caraballo, F. Cilleruelo. Fuel optimization strategy for hydrogen fuel cell range extender vehicles applying genetic algorithms. Renew. Sustain. Energy Rev., Vol. 81, Jan. 2018, Pages 655-668.

[10]. H. Hemi, J. Ghouili, A. Cheriti. Combination of Markov chain and optimal control solved by Pontryagin's Minimum Principle for a fuel cell/supercapacitor vehicle. Energy Conv. Manag.. Vol 91, Page: 387-393, Feb 2015.

[11]. S. Onori, L. Tribioli. Adaptive Pontryagin's Minimum Principle supervisory controller design for the plug-in hybrid GM Chevrolet Volt. Appl. Energy, Vol. 147, Jun. 2015, Pages 224-234.

[12]. W. Zhang, J. Li, L. Xu, M. Ouyang, Optimization for a fuel cell/battery/capacity tram with equivalent consumption minimization strategy, Energy Conv. Manag., Vol. 134, 2017, Pages 59-69.

[13]. H. Li, A. Ravey, A. N'Diaye, A. Djerdir. A novel equivalent consumption minimization strategy for hybrid electric vehicle powered by fuel cell, battery and supercapacitor. J. Power Sources, Vol. 395, Aug. 2018, Pages 262-270.

[14]. G. Li, J. Zhang, H. He. Battery SOC constraint comparison for predictive energy management of plug-in hybrid electric bus. Appl. Energy, Vol. 194, May 2017, Pages 578-587.

[15]. S. Xie, X. Hu, Z. Xin, L. Li. Time-Efficient Stochastic Model Predictive Energy Management for a Plug-In Hybrid Electric Bus With an Adaptive Reference State-of-Charge Advisory. IEEE Trans. Veh. Technol., Vol. 67, No.7, Jul. 2018.

[16]. Y. Liu, J. Li, Z. Chen, D. Qin, Y. Zhang. Research on a multi-objective hierarchical prediction energy management strategy for range extended fuel cell vehicles. J. Power Sources, Vol. 429, Jul. 2019, Pages 55-66.

[17]. Y. Zhou, A. Ravey, MC. Péra, Multi-mode predictive energy management for fuel cell hybrid electric vehicles using Markov driving pattern recognizer, Applied Energy, Vol. 258, 2020, 114057.

679 [18]. D. Hou, Q. Sun, C. Bao, X. Cheng, H. Guo, Y. Zhao. An all-in-one design method for plug-in hybrid electric buses considering uncertain 
[19]. Y. Zhou, H. Li, A. Ravey, MC. Péra, An integrated predictive energy management for light-duty range-extended plug-in fuel cell electric vehicle, Journal of Power Sources, Vol. 451, 2020, 227780.

[20]. M. Sivertsson, L. Eriksson. Design and evaluation of energy management using map-based ECMS for the PHEV benchmark. Oil \& Gas Science and Technology, Vol. 70, No. 1, Jan 2014.

[21]. C. Sun, S. J. Moura, X. Hu, J. K. Hedrick, F. Sun. Dynamic Traffic Feedback Data Enabled Energy Management in Plug-in Hybrid Electric Vehicles. IEEE Trans. Control Syst. Technol., Vol. 23, No. 3, May 2015.

[22]. H. Tian, Z. Lu, X. Wang, X. Zhang, Y. Huang, G. Tian. A length ratio based neural network energy management strategy for online control of plug-in hybrid electric city bus. Appl. Energy, Vol. 177, Sep 2016, Pages 71-80.

[23]. J. G, H. He, J. Peng, N. Zhou. A novel MPC-based adaptive energy management strategy in plug-in hybrid electric vehicles. Energy, Vol. 175, May 2019, Pages 378-392.

[24]. H. Tian, X. Wang, Z. Lu, Y. Huang, G. Tian. Adaptive Fuzzy Logic Energy Management Strategy Based on Reasonable SOC Reference Curve for Online Control of Plug-in Hybrid Electric City Bus. IEEE trans. Intell. Transp. Syst., Vol. 19, No. 5, May 2018.

[25]. H. Tian, S. Li, X. Wang, Y. Huang, G. Tian. Data-driven hierarchical control for online energy management of plug-in hybrid electric city bus. Energy, Vol. 142, Jan. 2018, Pages 55-67.

[26]. M. Montazeri-Gh, Z. Pourbafarani, Near-Optimal SOC Trajectory for Traffic-Based Adaptive PHEV Control Strategy. IEEE Trans. Veh. Technol., Vol. 66, No. 11, Nov. 2017.

[27]. Y. Zhou. A. Ravey, MC. Péra, A survey on driving prediction techniques for predictive energy management of plug-in hybrid electric vehicles, J. Power Sources, Volume 412, 2019, Pages 480-495.

[28]. A. Ravey, N. Watrin, B. Blunier, D. Bouquain, A. Miraoui, Energy-Source-Sizing Methodology for Hybrid Fuel Cell Vehicles Based on Statistical Description of Driving Cycles, IEEE Trans. Veh. Technol., Vol. 60, no. 9, pp. 4164-4174, Nov. 2011.

[29]. L. Guzzella, A. Sciarretta. Vehicle Propulsion Systems: Introduction to Modeling and Optimization. Berlin: Springer-Verlag, pp. 14-18, 2005 .

[30]. MC. Péra, D. Hissel, H. Gualous, C. Turpin. Electrochemical Components. John Wiley \& Sons, Inc. 2013.

[31]. D. Zhou, A. Ravey, A. Durra, F. Gao. A comparative study of extremum seeking methods applied to online energy management strategy of fuel cell hybrid electric vehicles. Energy Conv. Manag., Vol.151, Pages: 778-790, Nov. 2017.

[32]. V. H. Johnson. Battery performance models in ADVISOR. J. Power Sources. Vol. 110, Issue 2, Pages 321-329, Aug 2002.

[33]. D. P. Filev, I. Kolmanovsky, Generalized markov models for real-time modeling of continuous systems, IEEE Trans. Fuzzy Syst., vol. 22, no. 4, pp. 983-998, 2014.

[34]. I. V. Kolmanovsky, D. P. Filev. Stochastic optimal control of systems with soft constraints and opportunities for automotive applications. 2009 IEEE Control Applications, (CCA) \& Intelligent Control, (ISIC).

[35]. P. Yi, X. Li, L. Yao, F. Fan, L. Peng, X. Lai, A lifetime prediction model for coated metallic bipolar plates in proton exchange membrane fuel cells, Energy Conv. Manag., Vol. 183, 2019, Pages 65-72.

[36]. C. Sun, X. Hu, S. J. Moura, F. Sun. Velocity Predictors for Predictive Energy Management in Hybrid Electric Vehicles. IEEE Trans. Control Syst. Technol., Vol. 23(3), May 2015. 